X AXIS Descriptives

\begin{tabular}{|c|c|c|c|c|}
\hline & & & Statistic & Std. Error \\
\hline \multirow[t]{13}{*}{11,12} & Mean & & ,0002313175 & ,0000582328 \\
\hline & \multirow{2}{*}{$\begin{array}{l}95 \% \text { Confidence Interval for } \\
\text { Mean }\end{array}$} & Lower Bound & ,0001166144 & \\
\hline & & Upper Bound & ,0003460205 & \\
\hline & $5 \%$ Trimmed Mean & & ,0002086020 & \\
\hline & Median & & ,0002007919 & \\
\hline & Variance & & ,000 & \\
\hline & Std. Deviation & &, 0009114869 & \\
\hline & Minimum & &,- 001998748 & \\
\hline & Maximum & & ,0030890283 & \\
\hline & Range & & ,0050877763 & \\
\hline & Interquartile Range & & ,0012657572 & \\
\hline & Skewness & & ,349 & ,156 \\
\hline & Kurtosis & &, 018 & ,310 \\
\hline \multirow[t]{13}{*}{13,14} & Mean & & ,0001915536 & ,0000536855 \\
\hline & \multirow{2}{*}{$\begin{array}{l}95 \% \text { Confidence Interval for } \\
\text { Mean }\end{array}$} & Lower Bound & ,0000858075 & \\
\hline & & Upper Bound & ,0002972997 & \\
\hline & $5 \%$ Trimmed Mean & &, 0001730037 & \\
\hline & Median & & ,0001438982 & \\
\hline & Variance & & ,000 & \\
\hline & Std. Deviation & & ,0008403103 & \\
\hline & Minimum & &,- 002019089 & \\
\hline & Maximum & &, 0026421448 & \\
\hline & Range & &, 0046612338 & \\
\hline & Interquartile Range & & ,0011691084 & \\
\hline & Skewness & & ,318 & , 156 \\
\hline & Kurtosis & &,- 077 & ,310 \\
\hline \multirow[t]{12}{*}{15,16} & Mean & &, 0001462272 &, 0000500013 \\
\hline & \multirow{2}{*}{$\begin{array}{l}95 \% \text { Confidence Interval for } \\
\text { Mean }\end{array}$} & Lower Bound & ,0000477379 & \\
\hline & & Upper Bound & ,0002447164 & \\
\hline & $5 \%$ Trimmed Mean & & ,0001317494 & \\
\hline & Median & & ,0000451162 & \\
\hline & Variance & &, 000 & \\
\hline & Std. Deviation & & ,0007826442 & \\
\hline & Minimum & &,- 002014102 & \\
\hline & Maximum & & ,0024831282 & \\
\hline & Range & & ,0044972299 & \\
\hline & Interquartile Range & & ,0010149443 & \\
\hline & Skewness & & ,294 & ,156 \\
\hline
\end{tabular}


X AXIS Descriptives

\begin{tabular}{|c|c|c|c|c|}
\hline & & & Statistic & Std. Error \\
\hline & Kurtosis & & , 100 & ,310 \\
\hline \multirow[t]{13}{*}{17,18} & Mean & &, 0001248311 & ,0000481841 \\
\hline & \multirow{2}{*}{$\begin{array}{l}95 \% \text { Confidence Interval for } \\
\text { Mean }\end{array}$} & Lower Bound & ,0000299213 & \\
\hline & & Upper Bound & ,0002197409 & \\
\hline & $5 \%$ Trimmed Mean & & ,0001123266 & \\
\hline & Median & & ,0000579852 & \\
\hline & Variance & &, 000 & \\
\hline & Std. Deviation & & ,0007542000 & \\
\hline & Minimum & &,- 001791725 & \\
\hline & Maximum & & ,0025014192 & \\
\hline & Range & & ,0042931438 & \\
\hline & Interquartile Range & & ,0010380833 & \\
\hline & Skewness & & ,292 & ,156 \\
\hline & Kurtosis & & ,291 & ,310 \\
\hline \multirow[t]{13}{*}{19,20} & Mean & & ,0001310097 & ,0000476674 \\
\hline & \multirow{2}{*}{$\begin{array}{l}95 \% \text { Confidence Interval for } \\
\text { Mean }\end{array}$} & Lower Bound & ,0000371176 & \\
\hline & & Upper Bound & ,0002249017 & \\
\hline & $5 \%$ Trimmed Mean & & ,0001249323 & \\
\hline & Median & &, 0000865113 & \\
\hline & Variance & &, 000 & \\
\hline & Std. Deviation & & ,0007461124 & \\
\hline & Minimum & &,- 001892891 & \\
\hline & Maximum & & ,0023488205 & \\
\hline & Range & & 0042417115 & \\
\hline & Interquartile Range & & ,0010573286 & \\
\hline & Skewness & &, 174 & ,156 \\
\hline & Kurtosis & &,- 062 & ,310 \\
\hline \multirow[t]{11}{*}{21,22} & Mean & &, 0001433855 & ,0000504593 \\
\hline & \multirow{2}{*}{$\begin{array}{l}95 \% \text { Confidence Interval for } \\
\text { Mean }\end{array}$} & Lower Bound & ,0000439941 & \\
\hline & & Upper Bound & ,0002427770 & \\
\hline & $5 \%$ Trimmed Mean & & ,0001382899 & \\
\hline & Median & & ,0001159466 & \\
\hline & Variance & &, 000 & \\
\hline & Std. Deviation & & ,0007898135 & \\
\hline & Minimum & &,- 001945942 & \\
\hline & Maximum & & ,0024383784 & \\
\hline & Range & & ,0043843203 & \\
\hline & Interquartile Range & & ,0011035405 & \\
\hline
\end{tabular}


X AXIS Descriptives

\begin{tabular}{|c|c|c|c|c|}
\hline & & & Statistic & Std. Error \\
\hline & \multicolumn{2}{|l|}{ Skewness } & ,129 & ,156 \\
\hline & \multicolumn{2}{|l|}{ Kurtosis } &, 032 & ,310 \\
\hline \multirow[t]{13}{*}{23,24} & \multicolumn{2}{|l|}{ Mean } & ,0002096760 &, 0000536118 \\
\hline & \multirow{2}{*}{$\begin{array}{l}95 \% \text { Confidence Interval for } \\
\text { Mean }\end{array}$} & Lower Bound & ,0001040750 & \\
\hline & & Upper Bound &, 0003152771 & \\
\hline & \multicolumn{2}{|l|}{$5 \%$ Trimmed Mean } & ,0002085495 & \\
\hline & \multicolumn{2}{|l|}{ Median } &, 0001674354 & \\
\hline & \multicolumn{2}{|l|}{ Variance } &, 000 & \\
\hline & \multicolumn{2}{|l|}{ Std. Deviation } & ,0008391579 & \\
\hline & \multicolumn{2}{|l|}{ Minimum } &,- 002242157 & \\
\hline & \multicolumn{2}{|l|}{ Maximum } & ,0025418873 & \\
\hline & \multicolumn{2}{|l|}{ Range } &, 0047840446 & \\
\hline & \multicolumn{2}{|l|}{ Interquartile Range } &, 0011129330 & \\
\hline & \multicolumn{2}{|l|}{ Skewness } & ,032 & ,156 \\
\hline & \multicolumn{2}{|l|}{ Kurtosis } & ,132 & ,310 \\
\hline \multirow[t]{13}{*}{25,26} & \multicolumn{2}{|l|}{ Mean } & ,0002945792 & ,0000592178 \\
\hline & \multirow{2}{*}{$\begin{array}{l}95 \% \text { Confidence Interval for } \\
\text { Mean }\end{array}$} & Lower Bound & ,0001779358 & \\
\hline & & Upper Bound &, 0004112225 & \\
\hline & \multicolumn{2}{|l|}{$5 \%$ Trimmed Mean } &, 0002867914 & \\
\hline & \multicolumn{2}{|l|}{ Median } & ,0002200923 & \\
\hline & \multicolumn{2}{|l|}{ Variance } &, 000 & \\
\hline & \multicolumn{2}{|l|}{ Std. Deviation } &, 0009269056 & \\
\hline & \multicolumn{2}{|l|}{ Minimum } &,- 002786896 & \\
\hline & \multicolumn{2}{|l|}{ Maximum } & ,0028084091 & \\
\hline & \multicolumn{2}{|l|}{ Range } &, 0055953049 & \\
\hline & \multicolumn{2}{|l|}{ Interquartile Range } & ,0012749785 & \\
\hline & \multicolumn{2}{|l|}{ Skewness } &, 037 & ,156 \\
\hline & Kurtosis & & ,313 & ,310 \\
\hline 27,28 & Mean & &, 0003601511 &, 0000618856 \\
\hline & $95 \%$ Confidence Interval for & Lower Bound & ,0002382528 & \\
\hline & Mean & Upper Bound & ,0004820493 & \\
\hline & $5 \%$ Trimmed Mean & &, 0003554005 & \\
\hline & Median & &, 0003345247 & \\
\hline & Variance & &, 000 & \\
\hline & Std. Deviation & &, 0009686636 & \\
\hline & Minimum & &,- 002892938 & \\
\hline & Maximum & &, 0032073878 & \\
\hline & Range & & ,0061003258 & \\
\hline
\end{tabular}


X AXIS Descriptives

\begin{tabular}{|c|c|c|c|c|}
\hline & & & Statistic & Std. Error \\
\hline & \multicolumn{2}{|l|}{ Interquartile Range } & ,0014482753 & \\
\hline & \multicolumn{2}{|l|}{ Skewness } &,- 025 & , 156 \\
\hline & \multicolumn{2}{|l|}{ Kurtosis } & ,228 & ,310 \\
\hline \multirow[t]{13}{*}{29,30} & \multicolumn{2}{|l|}{ Mean } & ,0004260654 & ,0000626813 \\
\hline & \multirow{2}{*}{$\begin{array}{l}\text { 95\% Confidence Interval for } \\
\text { Mean }\end{array}$} & Lower Bound & ,0003025998 & \\
\hline & & Upper Bound & ,0005495309 & \\
\hline & \multicolumn{2}{|l|}{$5 \%$ Trimmed Mean } & ,0004201453 & \\
\hline & \multicolumn{2}{|l|}{ Median } & ,0004115212 & \\
\hline & \multicolumn{2}{|l|}{ Variance } &, 000 & \\
\hline & \multicolumn{2}{|l|}{ Std. Deviation } & ,0009811179 & \\
\hline & \multicolumn{2}{|l|}{ Minimum } &,- 003052044 & \\
\hline & \multicolumn{2}{|l|}{ Maximum } & ,0031976057 & \\
\hline & \multicolumn{2}{|l|}{ Range } & ,0062496501 & \\
\hline & \multicolumn{2}{|l|}{ Interquartile Range } & ,0014207869 & \\
\hline & \multicolumn{2}{|l|}{ Skewness } &,- 029 & ,156 \\
\hline & \multicolumn{2}{|l|}{ Kurtosis } & ,427 & ,310 \\
\hline \multirow[t]{13}{*}{31,32} & \multicolumn{2}{|l|}{ Mean } & ,0005016958 & ,0000629827 \\
\hline & \multirow{2}{*}{$\begin{array}{l}95 \% \text { Confidence Interval for } \\
\text { Mean }\end{array}$} & Lower Bound & ,0003776365 & \\
\hline & & Upper Bound &, 0006257550 & \\
\hline & \multicolumn{2}{|l|}{ 5\% Trimmed Mean } & ,0004959944 & \\
\hline & \multicolumn{2}{|l|}{ Median } &, 0005272585 & \\
\hline & \multicolumn{2}{|l|}{ Variance } & ,000 & \\
\hline & \multicolumn{2}{|l|}{ Std. Deviation } & ,0009858359 & \\
\hline & \multicolumn{2}{|l|}{ Minimum } &,- 003020961 & \\
\hline & \multicolumn{2}{|l|}{ Maximum } & ,0033883472 & \\
\hline & \multicolumn{2}{|l|}{ Range } & ,0064093083 & \\
\hline & \multicolumn{2}{|l|}{ Interquartile Range } & ,0012731669 & \\
\hline & Skewness & &,- 063 & ,156 \\
\hline & Kurtosis & & ,761 & ,310 \\
\hline 33,34 & Mean & & ,0005107779 &, 0000589451 \\
\hline & $95 \%$ Confidence Interval for & Lower Bound & ,0003946717 & \\
\hline & Mean & Upper Bound &, 0006268840 & \\
\hline & $5 \%$ Trimmed Mean & &, 0005028451 & \\
\hline & Median & &, 0005306640 & \\
\hline & Variance & &, 000 & \\
\hline & Std. Deviation & & ,0009226367 & \\
\hline & Minimum & &,- 002460835 & \\
\hline & Maximum & & ,0032758157 & \\
\hline
\end{tabular}


X AXIS Descriptives

\begin{tabular}{|c|c|c|c|c|}
\hline & & & Statistic & Std. Error \\
\hline & \multicolumn{2}{|l|}{ Range } & ,0057366509 & \\
\hline & \multicolumn{2}{|l|}{ Interquartile Range } &, 0012161436 & \\
\hline & \multicolumn{2}{|l|}{ Skewness } & 057 & ,156 \\
\hline & \multicolumn{2}{|l|}{ Kurtosis } & ,499 & ,310 \\
\hline \multirow[t]{13}{*}{35,36} & \multicolumn{2}{|l|}{ Mean } & ,0005099598 & ,0000556038 \\
\hline & \multirow{2}{*}{$\begin{array}{l}95 \% \text { Confidence Interval for } \\
\text { Mean }\end{array}$} & Lower Bound &, 0004004350 & \\
\hline & & Upper Bound &, 0006194846 & \\
\hline & \multicolumn{2}{|l|}{$5 \%$ Trimmed Mean } &, 0005075355 & \\
\hline & \multicolumn{2}{|l|}{ Median } &, 0005035220 & \\
\hline & \multicolumn{2}{|l|}{ Variance } &, 000 & \\
\hline & \multicolumn{2}{|l|}{ Std. Deviation } & ,0008703379 & \\
\hline & \multicolumn{2}{|l|}{ Minimum } &,- 002554899 & \\
\hline & \multicolumn{2}{|l|}{ Maximum } &, 0033421806 & \\
\hline & \multicolumn{2}{|l|}{ Range } & ,0058970795 & \\
\hline & \multicolumn{2}{|l|}{ Interquartile Range } & ,0011433883 & \\
\hline & \multicolumn{2}{|l|}{ Skewness } &, 016 & ,156 \\
\hline & \multicolumn{2}{|l|}{ Kurtosis } &, 581 & ,310 \\
\hline \multirow[t]{13}{*}{37,38} & \multicolumn{2}{|l|}{ Mean } & ,0004126229 &, 0000462770 \\
\hline & \multirow{2}{*}{$\begin{array}{l}95 \% \text { Confidence Interval for } \\
\text { Mean }\end{array}$} & Lower Bound & ,0003214695 & \\
\hline & & Upper Bound & ,0005037763 & \\
\hline & \multicolumn{2}{|l|}{$5 \%$ Trimmed Mean } & ,0004095575 & \\
\hline & \multicolumn{2}{|l|}{ Median } & ,0004056691 & \\
\hline & \multicolumn{2}{|l|}{ Variance } &, 000 & \\
\hline & \multicolumn{2}{|l|}{ Std. Deviation } & ,0007243498 & \\
\hline & \multicolumn{2}{|l|}{ Minimum } &,- 002258597 & \\
\hline & \multicolumn{2}{|l|}{ Maximum } & ,0026842525 & \\
\hline & \multicolumn{2}{|l|}{ Range } & ,0049428491 & \\
\hline & Interquartile Range & & ,0009940134 & \\
\hline & Skewness & &,- 030 & ,156 \\
\hline & Kurtosis & & ,329 & ,310 \\
\hline 39,40 & Mean & &, 0002941511 &, 0000354241 \\
\hline & $95 \%$ Confidence Interval for & Lower Bound & ,0002243751 & \\
\hline & IVlean & Upper Bound & ,0003639272 & \\
\hline & $5 \%$ Trimmed Mean & & ,0002934700 & \\
\hline & Median & & ,0002778220 & \\
\hline & Variance & &, 000 & \\
\hline & Std. Deviation & &, 0005544750 & \\
\hline & Minimum & &,- 001584039 & \\
\hline
\end{tabular}


X AXIS Descriptives

\begin{tabular}{|c|c|c|c|c|}
\hline & & & Statistic & Std. Error \\
\hline & \multicolumn{2}{|l|}{ Maximum } & ,0018851274 & \\
\hline & \multicolumn{2}{|l|}{ Range } & ,0034691662 & \\
\hline & \multicolumn{2}{|l|}{ Interquartile Range } & ,0007555621 & \\
\hline & \multicolumn{2}{|l|}{ Skewness } &,- 008 & , 156 \\
\hline & \multicolumn{2}{|l|}{ Kurtosis } & ,139 & ,310 \\
\hline \multirow[t]{13}{*}{41,42} & \multicolumn{2}{|l|}{ Mean } & ,0001382077 & ,0000202430 \\
\hline & \multirow{2}{*}{$\begin{array}{l}95 \% \text { Confidence Interval for } \\
\text { Mean }\end{array}$} & Lower Bound & ,0000983343 & \\
\hline & & Upper Bound & ,0001780810 & \\
\hline & \multicolumn{2}{|l|}{$5 \%$ Trimmed Mean } & ,0001432808 & \\
\hline & \multicolumn{2}{|l|}{ Median } & ,0001402215 & \\
\hline & \multicolumn{2}{|l|}{ Variance } &, 000 & \\
\hline & \multicolumn{2}{|l|}{ Std. Deviation } & ,0003168533 & \\
\hline & \multicolumn{2}{|l|}{ Minimum } &,- 001189085 & \\
\hline & \multicolumn{2}{|l|}{ Maximum } & ,0008735133 & \\
\hline & \multicolumn{2}{|l|}{ Range } & ,0020625983 & \\
\hline & \multicolumn{2}{|l|}{ Interquartile Range } & ,0004269287 & \\
\hline & \multicolumn{2}{|l|}{ Skewness } &,- 367 & , 156 \\
\hline & \multicolumn{2}{|l|}{ Kurtosis } & ,821 & ,310 \\
\hline \multirow[t]{13}{*}{43,44} & \multicolumn{2}{|l|}{ Mean } &,- 000227620 &, 0001068576 \\
\hline & \multirow{2}{*}{$\begin{array}{l}95 \% \text { Confidence Interval for } \\
\text { Mean }\end{array}$} & Lower Bound &,- 000438101 & \\
\hline & & Upper Bound &,- 000017139 & \\
\hline & \multicolumn{2}{|l|}{$5 \%$ Trimmed Mean } &,- 000248089 & \\
\hline & \multicolumn{2}{|l|}{ Median } &,- 000391829 & \\
\hline & \multicolumn{2}{|l|}{ Variance } &, 000 & \\
\hline & \multicolumn{2}{|l|}{ Std. Deviation } & ,0016725866 & \\
\hline & \multicolumn{2}{|l|}{ Minimum } &,- 003994682 & \\
\hline & \multicolumn{2}{|l|}{ Maximum } & ,0045002192 & \\
\hline & Range & & ,0084949016 & \\
\hline & Interquartile Range & & ,0025165858 & \\
\hline & Skewness & & ,196 & ,156 \\
\hline & Kurtosis & &,- 406 & ,310 \\
\hline 45,46 & Mean & &,- 000249842 & ,0000860082 \\
\hline & $95 \%$ Confidence Interval for & Lower Bound &,- 000419255 & \\
\hline & Iviean & Upper Bound &,- 000080428 & \\
\hline & 5\% Trimmed Mean & &,- 000212150 & \\
\hline & Median & &,- 000220688 & \\
\hline & Variance & & ,000 & \\
\hline & Std. Deviation & & ,0013462420 & \\
\hline
\end{tabular}


X AXIS Descriptives

\begin{tabular}{|c|c|c|c|c|}
\hline & & & Statistic & Std. Error \\
\hline & \multicolumn{2}{|l|}{ Minimum } &,- 004528986 & \\
\hline & \multicolumn{2}{|l|}{ Maximum } & ,0027614867 & \\
\hline & \multicolumn{2}{|l|}{ Range } & ,0072904725 & \\
\hline & \multicolumn{2}{|l|}{ Interquartile Range } & ,0017675688 & \\
\hline & \multicolumn{2}{|l|}{ Skewness } &,- 379 & ,156 \\
\hline & \multicolumn{2}{|l|}{ Kurtosis } &, 130 & ,310 \\
\hline \multirow[t]{13}{*}{47,48} & \multicolumn{2}{|l|}{ Mean } &,- 000240486 & ,0000813958 \\
\hline & \multirow{2}{*}{$\begin{array}{l}95 \% \text { Confidence Interval for } \\
\text { Mean }\end{array}$} & Lower Bound &,- 000400814 & \\
\hline & & Upper Bound &,- 000080158 & \\
\hline & \multicolumn{2}{|l|}{ 5\% Trimmed Mean } &,- 000201586 & \\
\hline & \multicolumn{2}{|l|}{ Median } &,- 000201562 & \\
\hline & \multicolumn{2}{|l|}{ Variance } &, 000 & \\
\hline & \multicolumn{2}{|l|}{ Std. Deviation } & ,0012740453 & \\
\hline & \multicolumn{2}{|l|}{ Minimum } &,- 004265590 & \\
\hline & \multicolumn{2}{|l|}{ Maximum } & ,0025691772 & \\
\hline & \multicolumn{2}{|l|}{ Range } & ,0068347674 & \\
\hline & \multicolumn{2}{|l|}{ Interquartile Range } & ,0016904216 & \\
\hline & \multicolumn{2}{|l|}{ Skewness } &,- 356 & ,156 \\
\hline & \multicolumn{2}{|l|}{ Kurtosis } &, 027 & ,310 \\
\hline \multirow[t]{13}{*}{49,50} & \multicolumn{2}{|l|}{ Mean } &,- 000131140 &, 0000729641 \\
\hline & \multirow{2}{*}{$\begin{array}{l}95 \% \text { Confidence Interval for } \\
\text { Mean }\end{array}$} & Lower Bound &,- 000274860 & \\
\hline & & Upper Bound &, 0000125798 & \\
\hline & \multicolumn{2}{|l|}{ 5\% Trimmed Mean } &,- 000131671 & \\
\hline & \multicolumn{2}{|l|}{ Median } &,- 000151029 & \\
\hline & \multicolumn{2}{|l|}{ Variance } &, 000 & \\
\hline & \multicolumn{2}{|l|}{ Std. Deviation } &, 0011420684 & \\
\hline & \multicolumn{2}{|l|}{ Minimum } &,- 003556603 & \\
\hline & Maximum & &, 0029439335 & \\
\hline & Range & &, 0065005368 & \\
\hline & Interquartile Range & &, 0015399570 & \\
\hline & Skewness & &,- 030 & ,156 \\
\hline & Kurtosis & &,- 199 & ,310 \\
\hline 51,52 & Mean & & ,0000275336 & ,0000716005 \\
\hline & $95 \%$ Confidence Interval for & Lower Bound &,- 000113500 & \\
\hline & IVlean & Upper Bound & ,0001685675 & \\
\hline & $5 \%$ Trimmed Mean & & ,0000204484 & \\
\hline & Median & & ,0000500568 & \\
\hline & Variance & &, 000 & \\
\hline
\end{tabular}


X AXIS Descriptives

\begin{tabular}{|c|c|c|c|c|}
\hline & & & Statistic & Std. Error \\
\hline & \multicolumn{2}{|l|}{ Std. Deviation } & ,0011207249 & \\
\hline & \multicolumn{2}{|l|}{ Minimum } &,- 003001613 & \\
\hline & \multicolumn{2}{|l|}{ Maximum } & ,0042898205 & \\
\hline & \multicolumn{2}{|l|}{ Range } & ,0072914334 & \\
\hline & \multicolumn{2}{|l|}{ Interquartile Range } & ,0014562022 & \\
\hline & \multicolumn{2}{|l|}{ Skewness } & ,138 & ,156 \\
\hline & \multicolumn{2}{|l|}{ Kurtosis } & ,495 & ,310 \\
\hline \multirow[t]{13}{*}{53,54} & \multicolumn{2}{|l|}{ Mean } &, 0000435064 & ,0000689935 \\
\hline & \multirow{2}{*}{$\begin{array}{l}\text { 95\% Confidence Interval for } \\
\text { Mean }\end{array}$} & Lower Bound &,- 000092392 & \\
\hline & & Upper Bound & ,0001794053 & \\
\hline & \multicolumn{2}{|l|}{$5 \%$ Trimmed Mean } &, 0000446780 & \\
\hline & \multicolumn{2}{|l|}{ Median } & ,0000789240 & \\
\hline & \multicolumn{2}{|l|}{ Variance } &, 000 & \\
\hline & \multicolumn{2}{|l|}{ Std. Deviation } & ,0010799190 & \\
\hline & \multicolumn{2}{|l|}{ Minimum } &,- 003102189 & \\
\hline & \multicolumn{2}{|l|}{ Maximum } & ,0044328180 & \\
\hline & \multicolumn{2}{|l|}{ Range } & ,0075350072 & \\
\hline & \multicolumn{2}{|l|}{ Interquartile Range } & ,0014607731 & \\
\hline & \multicolumn{2}{|l|}{ Skewness } & 072 & ,156 \\
\hline & \multicolumn{2}{|l|}{ Kurtosis } & ,795 & ,310 \\
\hline \multirow[t]{13}{*}{55,56} & \multicolumn{2}{|l|}{ Mean } &,- 000105380 & ,0000612265 \\
\hline & \multirow{2}{*}{$\begin{array}{l}\text { 95\% Confidence Interval for } \\
\text { Mean }\end{array}$} & Lower Bound &,- 000225979 & \\
\hline & & Upper Bound & ,0000152204 & \\
\hline & \multicolumn{2}{|l|}{$5 \%$ Trimmed Mean } &,- 000105584 & \\
\hline & \multicolumn{2}{|l|}{ Median } &,- 000073806 & \\
\hline & \multicolumn{2}{|l|}{ Variance } &, 000 & \\
\hline & \multicolumn{2}{|l|}{ Std. Deviation } & ,0009583463 & \\
\hline & Minimum & &,- 003042522 & \\
\hline & Maximum & & ,0027612225 & \\
\hline & Range & & ,0058037449 & \\
\hline & Interquartile Range & & ,0011672172 & \\
\hline & Skewness & &,- 031 & , 156 \\
\hline & Kurtosis & & ,226 & ,310 \\
\hline 57,58 & Mean & &,- 000223161 & ,0000766808 \\
\hline & $95 \%$ Confidence Interval for & Lower Bound &,- 000374202 & \\
\hline & IVlean & Upper Bound &,- 000072120 & \\
\hline & $5 \%$ Trimmed Mean & &,- 000207368 & \\
\hline & Median & &,- 000207827 & \\
\hline
\end{tabular}


X AXIS Descriptives

\begin{tabular}{|c|c|c|c|c|}
\hline & & & Statistic & Std. Error \\
\hline & \multicolumn{2}{|l|}{ Variance } &, 000 & \\
\hline & \multicolumn{2}{|l|}{ Std. Deviation } & ,0012002451 & \\
\hline & \multicolumn{2}{|l|}{ Minimum } &,- 003639461 & \\
\hline & \multicolumn{2}{|l|}{ Maximum } & ,0027072182 & \\
\hline & \multicolumn{2}{|l|}{ Range } & ,0063466790 & \\
\hline & \multicolumn{2}{|l|}{ Interquartile Range } & ,0014361590 & \\
\hline & \multicolumn{2}{|l|}{ Skewness } &,- 171 & ,156 \\
\hline & \multicolumn{2}{|l|}{ Kurtosis } &, 127 & ,310 \\
\hline \multirow[t]{13}{*}{59,60} & \multicolumn{2}{|l|}{ Mean } &,- 000269791 & ,0000871822 \\
\hline & \multirow{2}{*}{$\begin{array}{l}95 \% \text { Confidence Interval for } \\
\text { Mean }\end{array}$} & Lower Bound &,- 000441517 & \\
\hline & & Upper Bound &,- 000098066 & \\
\hline & \multicolumn{2}{|l|}{$5 \%$ Trimmed Mean } &,- 000252051 & \\
\hline & \multicolumn{2}{|l|}{ Median } &,- 000296667 & \\
\hline & \multicolumn{2}{|l|}{ Variance } &, 000 & \\
\hline & \multicolumn{2}{|l|}{ Std. Deviation } &, 0013646165 & \\
\hline & \multicolumn{2}{|l|}{ Minimum } &,- 004166241 & \\
\hline & \multicolumn{2}{|l|}{ Maximum } & ,0028322358 & \\
\hline & \multicolumn{2}{|l|}{ Range } & ,0069984763 & \\
\hline & \multicolumn{2}{|l|}{ Interquartile Range } & ,0017918683 & \\
\hline & \multicolumn{2}{|l|}{ Skewness } &,- 172 & ,156 \\
\hline & \multicolumn{2}{|l|}{ Kurtosis } &,- 071 & ,310 \\
\hline \multirow[t]{13}{*}{61,62} & \multicolumn{2}{|l|}{ Mean } &,- 000220828 & ,0000639908 \\
\hline & \multirow{2}{*}{$\begin{array}{l}95 \% \text { Confidence Interval for } \\
\text { Mean }\end{array}$} & Lower Bound &,- 000346873 & \\
\hline & & Upper Bound &,- 000094783 & \\
\hline & \multicolumn{2}{|l|}{$5 \%$ Trimmed Mean } &,- 000217334 & \\
\hline & \multicolumn{2}{|l|}{ Median } &,- 000210821 & \\
\hline & \multicolumn{2}{|l|}{ Variance } &, 000 & \\
\hline & Std. Deviation & & ,0010016151 & \\
\hline & Minimum & &,- 003054321 & \\
\hline & Maximum & & ,0021735364 & \\
\hline & Range & & ,0052278570 & \\
\hline & Interquartile Range & & ,0013460837 & \\
\hline & Skewness & &,- 031 & , 156 \\
\hline & Kurtosis & &,- 238 & ,310 \\
\hline 63,64 & Mean & & ,0000840169 & ,0000601878 \\
\hline & $95 \%$ Confidence Interval for & Lower Bound &,- 000034537 & \\
\hline & & Upper Bound & ,0002025709 & \\
\hline & $5 \%$ Trimmed Mean & & ,0000825053 & \\
\hline
\end{tabular}


X AXIS Descriptives

\begin{tabular}{|c|c|c|c|c|}
\hline & & & Statistic & Std. Error \\
\hline & \multicolumn{2}{|l|}{ Median } & ,0001039782 & \\
\hline & \multicolumn{2}{|l|}{ Variance } &, 000 & \\
\hline & \multicolumn{2}{|l|}{ Std. Deviation } & ,0009420885 & \\
\hline & \multicolumn{2}{|l|}{ Minimum } &,- 002526809 & \\
\hline & \multicolumn{2}{|l|}{ Maximum } & ,0024284013 & \\
\hline & \multicolumn{2}{|l|}{ Range } & ,0049552099 & \\
\hline & \multicolumn{2}{|l|}{ Interquartile Range } & ,0013464582 & \\
\hline & \multicolumn{2}{|l|}{ Skewness } &, 043 & ,156 \\
\hline & \multicolumn{2}{|l|}{ Kurtosis } &,- 238 & ,310 \\
\hline \multirow[t]{13}{*}{65,66} & \multicolumn{2}{|l|}{ Mean } &, 0002309626 & ,0000564059 \\
\hline & \multirow{2}{*}{$\begin{array}{l}95 \% \text { Confidence Interval for } \\
\text { Mean }\end{array}$} & Lower Bound & ,0001198580 & \\
\hline & & Upper Bound & ,0003420672 & \\
\hline & \multicolumn{2}{|l|}{$5 \%$ Trimmed Mean } & ,0002326745 & \\
\hline & \multicolumn{2}{|l|}{ Median } & ,0002891808 & \\
\hline & \multicolumn{2}{|l|}{ Variance } &, 000 & \\
\hline & \multicolumn{2}{|l|}{ Std. Deviation } & ,0008828919 & \\
\hline & \multicolumn{2}{|l|}{ Minimum } &,- 002371352 & \\
\hline & \multicolumn{2}{|l|}{ Maximum } & ,0026007027 & \\
\hline & \multicolumn{2}{|l|}{ Range } & ,0049720546 & \\
\hline & \multicolumn{2}{|l|}{ Interquartile Range } &, 0011540854 & \\
\hline & \multicolumn{2}{|l|}{ Skewness } &,- 075 & ,156 \\
\hline & \multicolumn{2}{|l|}{ Kurtosis } &, 035 & ,310 \\
\hline \multirow[t]{13}{*}{67,68} & \multicolumn{2}{|l|}{ Mean } & ,0000808358 & ,0000464558 \\
\hline & \multirow{2}{*}{$\begin{array}{l}95 \% \text { Confidence Interval for } \\
\text { Mean }\end{array}$} & Lower Bound &,- 000010670 & \\
\hline & & Upper Bound & 0001723413 & \\
\hline & \multicolumn{2}{|l|}{$5 \%$ Trimmed Mean } & ,0000808804 & \\
\hline & \multicolumn{2}{|l|}{ Median } & ,0001045974 & \\
\hline & Variance & &, 000 & \\
\hline & Std. Deviation & &, 0007271478 & \\
\hline & Minimum & &,- 001813749 & \\
\hline & Maximum & & ,0021678370 & \\
\hline & Range & &, 0039815858 & \\
\hline & Interquartile Range & & ,0009062305 & \\
\hline & Skewness & &, 051 & ,156 \\
\hline & Kurtosis & & ,039 & ,310 \\
\hline 69,70 & Mean & &,- 000144087 & ,0000393539 \\
\hline & $95 \%$ Confidence Interval for & Lower Bound &,- 000221604 & \\
\hline & IVlean & Upper Bound &,- 000066571 & \\
\hline
\end{tabular}


X AXIS Descriptives

\begin{tabular}{|c|c|c|c|c|}
\hline & & & Statistic & Std. Error \\
\hline & \multicolumn{2}{|l|}{$5 \%$ Trimmed Mean } &,- 000146619 & \\
\hline & \multicolumn{2}{|l|}{ Median } &,- 000144633 & \\
\hline & \multicolumn{2}{|l|}{ Variance } &, 000 & \\
\hline & \multicolumn{2}{|l|}{ Std. Deviation } & ,0006159855 & \\
\hline & \multicolumn{2}{|l|}{ Minimum } &,- 002087320 & \\
\hline & \multicolumn{2}{|l|}{ Maximum } & ,0018277232 & \\
\hline & \multicolumn{2}{|l|}{ Range } & ,0039150431 & \\
\hline & \multicolumn{2}{|l|}{ Interquartile Range } & ,0007945638 & \\
\hline & \multicolumn{2}{|l|}{ Skewness } &,- 019 & , 156 \\
\hline & \multicolumn{2}{|l|}{ Kurtosis } &, 526 & ,310 \\
\hline \multirow[t]{13}{*}{71,72} & \multicolumn{2}{|l|}{ Mean } & ,0000221871 & ,0000468567 \\
\hline & \multirow{2}{*}{$\begin{array}{l}95 \% \text { Confidence Interval for } \\
\text { Mean }\end{array}$} & Lower Bound &,- 000070108 & \\
\hline & & Upper Bound & ,0001144824 & \\
\hline & \multicolumn{2}{|l|}{$5 \%$ Trimmed Mean } & ,0000190753 & \\
\hline & \multicolumn{2}{|l|}{ Median } & ,0000249080 & \\
\hline & \multicolumn{2}{|l|}{ Variance } &, 000 & \\
\hline & \multicolumn{2}{|l|}{ Std. Deviation } &, 0007334238 & \\
\hline & \multicolumn{2}{|l|}{ Minimum } &,- 002253232 & \\
\hline & \multicolumn{2}{|l|}{ Maximum } & ,0021558484 & \\
\hline & \multicolumn{2}{|l|}{ Range } & ,0044090799 & \\
\hline & \multicolumn{2}{|l|}{ Interquartile Range } & ,0008999785 & \\
\hline & \multicolumn{2}{|l|}{ Skewness } &,- 020 & , 156 \\
\hline & \multicolumn{2}{|l|}{ Kurtosis } &, 525 & ,310 \\
\hline \multirow[t]{13}{*}{73,74} & \multicolumn{2}{|l|}{ Mean } & ,0001005493 & ,0000535998 \\
\hline & \multirow{2}{*}{$\begin{array}{l}95 \% \text { Confidence Interval for } \\
\text { Mean }\end{array}$} & Lower Bound &,- 000005028 & \\
\hline & & Upper Bound & ,0002061266 & \\
\hline & \multicolumn{2}{|l|}{$5 \%$ Trimmed Mean } & ,0001034964 & \\
\hline & \multicolumn{2}{|l|}{ Median } & ,0001332430 & \\
\hline & Variance & &, 000 & \\
\hline & Std. Deviation & & ,0008389694 & \\
\hline & Minimum & &,- 002383079 & \\
\hline & Maximum & & ,0025440950 & \\
\hline & Range & & ,0049271735 & \\
\hline & Interquartile Range & & ,0010222821 & \\
\hline & Skewness & &,- 071 & , 156 \\
\hline & Kurtosis & &, 470 &, 310 \\
\hline 75,76 & Mean & &,- 000004876 & ,0000564025 \\
\hline
\end{tabular}


X AXIS Descriptives

\begin{tabular}{|c|c|c|c|c|}
\hline & & & Statistic & Std. Error \\
\hline & \multirow{2}{*}{$\begin{array}{l}95 \% \text { Confidence Interval for } \\
\text { Mean }\end{array}$} & Lower Bound &,- 000115974 & \\
\hline & & Upper Bound & ,0001062220 & \\
\hline & $5 \%$ Trimmed Mean & & ,0000001969 & \\
\hline & Median & & ,0000165933 & \\
\hline & Variance & &, 000 & \\
\hline & Std. Deviation & & ,0008828390 & \\
\hline & Minimum & &,- 002710530 & \\
\hline & Maximum & & ,0023856571 & \\
\hline & Range & & ,0050961867 & \\
\hline & Interquartile Range & & ,0010985329 & \\
\hline & Skewness & &,- 154 & ,156 \\
\hline & Kurtosis & & ,255 & ,310 \\
\hline \multirow[t]{13}{*}{77,78} & Mean & &, 0002258512 & ,0000539680 \\
\hline & \multirow{2}{*}{$\begin{array}{l}95 \% \text { Confidence Interval for } \\
\text { Mean }\end{array}$} & Lower Bound & ,0001195487 & \\
\hline & & Upper Bound & ,0003321538 & \\
\hline & $5 \%$ Trimmed Mean & & ,0002267454 & \\
\hline & Median & & ,0002951020 & \\
\hline & Variance & &, 000 & \\
\hline & Std. Deviation & & 0008447324 & \\
\hline & Minimum & &,- 002146211 & \\
\hline & Maximum & & ,0027131992 & \\
\hline & Range & & ,0048594107 & \\
\hline & Interquartile Range & & ,0010189662 & \\
\hline & Skewness & &,- 004 & ,156 \\
\hline & Kurtosis & & ,426 & ,310 \\
\hline \multirow[t]{13}{*}{79,80} & Mean & &, 0002258512 &, 0000539680 \\
\hline & \multirow{2}{*}{$\begin{array}{l}95 \% \text { Confidence Interval for } \\
\text { Mean }\end{array}$} & Lower Bound & ,0001195487 & \\
\hline & & Upper Bound & ,0003321538 & \\
\hline & $5 \%$ Trimmed Mean & & ,0002267454 & \\
\hline & Median & & ,0002951020 & \\
\hline & Variance & &, 000 & \\
\hline & Std. Deviation & & ,0008447324 & \\
\hline & Minimum & &,- 002146211 & \\
\hline & Maximum & & ,0027131992 & \\
\hline & Range & & ,0048594107 & \\
\hline & Interquartile Range & & ,0010189662 & \\
\hline & Skewness & &,- 004 & ,156 \\
\hline & Kurtosis & & ,426 & ,310 \\
\hline
\end{tabular}


X AXIS Descriptives

\begin{tabular}{|c|c|c|c|c|}
\hline & & & Statistic & Std. Error \\
\hline \multirow[t]{13}{*}{81,82} & Mean & &,- 000055546 &, 0000188473 \\
\hline & \multirow{2}{*}{$\begin{array}{l}95 \% \text { Confidence Interval for } \\
\text { Mean }\end{array}$} & Lower Bound &,- 000092670 & \\
\hline & & Upper Bound &,- 000018422 & \\
\hline & $5 \%$ Trimmed Mean & &,- 000050972 & \\
\hline & Median & &,- 000049670 & \\
\hline & Variance & &, 000 & \\
\hline & Std. Deviation & &, 0002950070 & \\
\hline & Minimum & &,- 000945568 & \\
\hline & Maximum & &, 0007068617 & \\
\hline & Range & & ,0016524294 & \\
\hline & Interquartile Range & & ,0003659813 & \\
\hline & Skewness & &,- 218 & ,156 \\
\hline & Kurtosis & & ,320 & ,310 \\
\hline \multirow[t]{13}{*}{83,84} & Mean & &,- 000184536 &, 0000215416 \\
\hline & \multirow{2}{*}{$\begin{array}{l}95 \% \text { Confidence Interval for } \\
\text { Mean }\end{array}$} & Lower Bound &,- 000226968 & \\
\hline & & Upper Bound &,- 000142105 & \\
\hline & $5 \%$ Trimmed Mean & &,- 000179556 & \\
\hline & Median & &,- 000145836 & \\
\hline & Variance & &, 000 & \\
\hline & Std. Deviation & & ,0003371792 & \\
\hline & Minimum & &,- 001174734 & \\
\hline & Maximum & & ,0007306250 & \\
\hline & Range & & ,0019053586 & \\
\hline & Interquartile Range & &, 0004488236 & \\
\hline & Skewness & &,- 231 & ,156 \\
\hline & Kurtosis & &, 053 & ,310 \\
\hline \multirow[t]{12}{*}{85,86} & Mean & &,- 000220985 & ,0000296022 \\
\hline & \multirow{2}{*}{$\begin{array}{l}95 \% \text { Confidence Interval for } \\
\text { Mean }\end{array}$} & Lower Bound &,- 000279294 & \\
\hline & & Upper Bound &,- 000162677 & \\
\hline & $5 \%$ Trimmed Mean & &,- 000217546 & \\
\hline & Median & &,- 000180150 & \\
\hline & Variance & &, 000 & \\
\hline & Std. Deviation & & ,0004633479 & \\
\hline & Minimum & &,- 001540198 & \\
\hline & Maximum & & ,0010661347 & \\
\hline & Range & & ,0026063331 & \\
\hline & Interquartile Range & & ,0006321438 & \\
\hline & Skewness & &,- 146 & ,156 \\
\hline
\end{tabular}


X AXIS Descriptives

\begin{tabular}{|c|c|c|c|c|}
\hline & & & Statistic & Std. Error \\
\hline & Kurtosis & &, 061 & ,310 \\
\hline \multirow[t]{13}{*}{87,88} & Mean & &,- 000184325 &, 0000203642 \\
\hline & \multirow{2}{*}{$\begin{array}{l}95 \% \text { Confidence Interval for } \\
\text { Mean }\end{array}$} & Lower Bound &,- 000224437 & \\
\hline & & Upper Bound &,- 000144213 & \\
\hline & $5 \%$ Trimmed Mean & &,- 000181947 & \\
\hline & Median & &,- 000172365 & \\
\hline & Variance & &, 000 & \\
\hline & Std. Deviation & & ,0003187497 & \\
\hline & Minimum & &,- 001153601 & \\
\hline & Maximum & & ,0006871652 & \\
\hline & Range & & ,0018407664 & \\
\hline & Interquartile Range & &, 0004564656 & \\
\hline & Skewness & &,- 036 & , 156 \\
\hline & Kurtosis & &,- 200 & ,310 \\
\hline \multirow[t]{13}{*}{89,90} & Mean & &,- 000141736 & ,0000155714 \\
\hline & \multirow{2}{*}{$\begin{array}{l}95 \% \text { Confidence Interval for } \\
\text { Mean }\end{array}$} & Lower Bound &,- 000172407 & \\
\hline & & Upper Bound &,- 000111064 & \\
\hline & $5 \%$ Trimmed Mean & &,- 000144390 & \\
\hline & Median & &,- 000149724 & \\
\hline & Variance & &, 000 & \\
\hline & Std. Deviation & & ,0002437317 & \\
\hline & Minimum & &,- 000799569 & \\
\hline & Maximum & & ,0005082056 & \\
\hline & Range & & ,0013077749 & \\
\hline & Interquartile Range & & ,0003191285 & \\
\hline & Skewness & &, 087 & , 156 \\
\hline & Kurtosis & &,- 112 & ,310 \\
\hline \multirow[t]{11}{*}{91,92} & Mean & &, 0002124531 & ,0000540864 \\
\hline & \multirow{2}{*}{$\begin{array}{l}95 \% \text { Confidence Interval for } \\
\text { Mean }\end{array}$} & Lower Bound & ,0001059173 & \\
\hline & & Upper Bound & ,0003189889 & \\
\hline & $5 \%$ Trimmed Mean & &, 0002112602 & \\
\hline & Median & & ,0002320276 & \\
\hline & Variance & &, 000 & \\
\hline & Std. Deviation & &, 0008465856 & \\
\hline & Minimum & &,- 002077043 & \\
\hline & Maximum & & ,0026143419 & \\
\hline & Range & & ,0046913850 & \\
\hline & Interquartile Range & & ,0011741021 & \\
\hline
\end{tabular}


X AXIS Descriptives

\begin{tabular}{|c|c|c|c|c|}
\hline & & & Statistic & Std. Error \\
\hline & \multicolumn{2}{|l|}{ Skewness } &, 035 & , 156 \\
\hline & \multicolumn{2}{|l|}{ Kurtosis } &, 019 & ,310 \\
\hline \multirow[t]{13}{*}{93,94} & \multicolumn{2}{|l|}{ Mean } & ,0001298403 &, 0000341317 \\
\hline & \multirow{2}{*}{$\begin{array}{l}95 \% \text { Confidence Interval for } \\
\text { Mean }\end{array}$} & Lower Bound & ,0000626099 & \\
\hline & & Upper Bound & ,0001970708 & \\
\hline & \multicolumn{2}{|l|}{$5 \%$ Trimmed Mean } & ,0001280981 & \\
\hline & \multicolumn{2}{|l|}{ Median } & ,0001401190 & \\
\hline & \multicolumn{2}{|l|}{ Variance } &, 000 & \\
\hline & \multicolumn{2}{|l|}{ Std. Deviation } &, 0005342460 & \\
\hline & \multicolumn{2}{|l|}{ Minimum } &,- 001368469 & \\
\hline & \multicolumn{2}{|l|}{ Maximum } & ,0015462576 & \\
\hline & \multicolumn{2}{|l|}{ Range } & ,0029147262 & \\
\hline & \multicolumn{2}{|l|}{ Interquartile Range } & ,0007194778 & \\
\hline & \multicolumn{2}{|l|}{ Skewness } & ,020 & ,156 \\
\hline & \multicolumn{2}{|l|}{ Kurtosis } &, 034 & ,310 \\
\hline \multirow[t]{13}{*}{95,96} & \multicolumn{2}{|l|}{ Mean } & ,0001933900 & ,0000468171 \\
\hline & \multirow{2}{*}{$\begin{array}{l}95 \% \text { Confidence Interval for } \\
\text { Mean }\end{array}$} & Lower Bound & ,0001011727 & \\
\hline & & Upper Bound &, 0002856073 & \\
\hline & \multicolumn{2}{|l|}{$5 \%$ Trimmed Mean } &, 0001904234 & \\
\hline & \multicolumn{2}{|l|}{ Median } & ,0001924188 & \\
\hline & \multicolumn{2}{|l|}{ Variance } &, 000 & \\
\hline & \multicolumn{2}{|l|}{ Std. Deviation } &, 0007328041 & \\
\hline & \multicolumn{2}{|l|}{ Minimum } &,- 001935712 & \\
\hline & \multicolumn{2}{|l|}{ Maximum } & ,0022332070 & \\
\hline & \multicolumn{2}{|l|}{ Range } &, 0041689191 & \\
\hline & \multicolumn{2}{|l|}{ Interquartile Range } & ,0010029776 & \\
\hline & \multicolumn{2}{|l|}{ Skewness } &, 063 & ,156 \\
\hline & Kurtosis & & ,082 & ,310 \\
\hline 97,98 & Mean & &, 0000457027 &, 0000141166 \\
\hline & $95 \%$ Confidence Interval for & Lower Bound & ,0000178967 & \\
\hline & Mean & Upper Bound & ,0000735086 & \\
\hline & $5 \%$ Trimmed Mean & & ,0000454139 & \\
\hline & Median & &, 0000590383 & \\
\hline & Variance & &, 000 & \\
\hline & Std. Deviation & & ,0002209595 & \\
\hline & Minimum & &,- 000607129 & \\
\hline & Maximum & &, 0005995137 & \\
\hline & Range & & ,0012066431 & \\
\hline
\end{tabular}


X AXIS Descriptives

\begin{tabular}{|c|c|c|c|c|}
\hline & & & Statistic & Std. Error \\
\hline & \multicolumn{2}{|l|}{ Interquartile Range } & ,0003083711 & \\
\hline & \multicolumn{2}{|l|}{ Skewness } &,- 063 & ,156 \\
\hline & \multicolumn{2}{|l|}{ Kurtosis } &,- 215 & ,310 \\
\hline \multirow[t]{13}{*}{99,100} & Mean & & ,0000819929 & ,0000277677 \\
\hline & \multirow{2}{*}{$\begin{array}{l}95 \% \text { Confidence Interval for } \\
\text { Mean }\end{array}$} & Lower Bound &, 0000272980 & \\
\hline & & Upper Bound & ,0001366878 & \\
\hline & \multicolumn{2}{|l|}{$5 \%$ Trimmed Mean } & ,0000836778 & \\
\hline & \multicolumn{2}{|l|}{ Median } & ,0000991283 & \\
\hline & \multicolumn{2}{|l|}{ Variance } &, 000 & \\
\hline & \multicolumn{2}{|l|}{ Std. Deviation } & ,0004346326 & \\
\hline & \multicolumn{2}{|l|}{ Minimum } &,- 001076990 & \\
\hline & \multicolumn{2}{|l|}{ Maximum } & ,0012382431 & \\
\hline & \multicolumn{2}{|l|}{ Range } & ,0023152327 & \\
\hline & \multicolumn{2}{|l|}{ Interquartile Range } &, 0005594232 & \\
\hline & \multicolumn{2}{|l|}{ Skewness } &,- 074 & ,156 \\
\hline & \multicolumn{2}{|l|}{ Kurtosis } &,- 099 & ,310 \\
\hline \multirow[t]{13}{*}{101,102} & \multicolumn{2}{|l|}{ Mean } & ,0001412914 &, 0000399642 \\
\hline & \multirow{2}{*}{$\begin{array}{l}95 \% \text { Confidence Interval for } \\
\text { Mean }\end{array}$} & Lower Bound &, 0000625725 & \\
\hline & & Upper Bound & ,0002200103 & \\
\hline & \multicolumn{2}{|l|}{$5 \%$ Trimmed Mean } & ,0001415312 & \\
\hline & \multicolumn{2}{|l|}{ Median } & ,0001714503 & \\
\hline & \multicolumn{2}{|l|}{ Variance } &, 000 & \\
\hline & \multicolumn{2}{|l|}{ Std. Deviation } &, 0006255390 & \\
\hline & \multicolumn{2}{|l|}{ Minimum } &,- 001771270 & \\
\hline & \multicolumn{2}{|l|}{ Maximum } & ,0022756090 & \\
\hline & \multicolumn{2}{|l|}{ Range } &, 0040468788 & \\
\hline & \multicolumn{2}{|l|}{ Interquartile Range } & ,0007916464 & \\
\hline & \multicolumn{2}{|l|}{ Skewness } &, 046 & ,156 \\
\hline & Kurtosis & & ,396 & ,310 \\
\hline 103,104 & Mean & & ,0000807301 & ,0000285315 \\
\hline & $95 \%$ Confidence Interval for & Lower Bound & ,0000245307 & \\
\hline & Mean & Upper Bound &, 0001369295 & \\
\hline & $5 \%$ Trimmed Mean & & ,0000799971 & \\
\hline & Median & & ,0000955327 & \\
\hline & Variance & &, 000 & \\
\hline & Std. Deviation & & ,0004465883 & \\
\hline & Minimum & &,- 001076992 & \\
\hline & Maximum & & ,0012507966 & \\
\hline
\end{tabular}




\section{AXIS Descriptives}

\begin{tabular}{|c|c|c|c|c|}
\hline & & & Statistic & Std. Error \\
\hline & \multicolumn{2}{|l|}{ Range } & ,0023277890 & \\
\hline & \multicolumn{2}{|l|}{ Interquartile Range } & ,0006409496 & \\
\hline & \multicolumn{2}{|l|}{ Skewness } & ,025 & ,156 \\
\hline & \multicolumn{2}{|l|}{ Kurtosis } &,- 238 & ,310 \\
\hline \multirow[t]{13}{*}{105,106} & Mean & & ,0000508855 & ,0000242428 \\
\hline & \multirow{2}{*}{$\begin{array}{l}95 \% \text { Confidence Interval for } \\
\text { Mean }\end{array}$} & Lower Bound & ,0000031336 & \\
\hline & & Upper Bound & ,0000986374 & \\
\hline & \multicolumn{2}{|l|}{$5 \%$ Trimmed Mean } & ,0000537412 & \\
\hline & \multicolumn{2}{|l|}{ Median } & ,0000614648 & \\
\hline & \multicolumn{2}{|l|}{ Variance } &, 000 & \\
\hline & \multicolumn{2}{|l|}{ Std. Deviation } & ,0003794602 & \\
\hline & \multicolumn{2}{|l|}{ Minimum } &,- 000949343 & \\
\hline & \multicolumn{2}{|l|}{ Maximum } &, 0011415339 & \\
\hline & \multicolumn{2}{|l|}{ Range } & ,0020908773 & \\
\hline & \multicolumn{2}{|l|}{ Interquartile Range } & ,0004971880 & \\
\hline & \multicolumn{2}{|l|}{ Skewness } &,- 048 & ,156 \\
\hline & \multicolumn{2}{|l|}{ Kurtosis } & ,023 & ,310 \\
\hline
\end{tabular}

X AXIS Tests of Normality

\begin{tabular}{|c|c|c|c|c|c|c} 
& \multicolumn{2}{c}{ Kolmogorov-Smirnov } & \multicolumn{3}{c}{ Shapiro-Wilk } & Sig. \\
\hline 11,12 & Statistic & df & Sig. & Statistic & df &, 056 \\
\hline 13,14 &, 052 & 245 &, $200^{*}$ &, 989 & 245 &, 134 \\
\hline 15,16 &, 047 & 245 &, $200^{*}$ &, 991 & 245 &, 196 \\
\hline 17,18 &, 061 & 245 &, 028 &, 992 & 245 &, 146 \\
\hline 19,20 &, 046 & 245 &, $200^{*}$ &, 991 & 245 &, 463 \\
\hline 21,22 &, 044 & 245 &, $200^{*}$ &, 994 & 245 &, 526 \\
\hline 23,24 &, 040 & 245 &, $200^{*}$ &, 995 & 245 &, 514 \\
\hline 25,26 &, 037 & 245 &, $200^{*}$ &, 994 & 245 &, 434 \\
\hline 27,28 &, 037 & 245 &, $200^{*}$ &, 994 & 245 &, 616 \\
\hline 29,30 &, 039 & 245 &, $200^{*}$ &, 995 & 245 &, 231 \\
\hline 31,32 &, 040 & 245 &, $200^{*}$ &, 992 & 245 &, 071 \\
\hline 33,34 &, 043 & 245 &, $200^{*}$ &, 989 & 245 &, 478 \\
\hline 35,36 &, 029 & 245 &, $200^{*}$ &, 994 & 245 &, 821 \\
\hline 37,38 &, 030 & 245 &, $200^{*}$ &, 996 & 245 &, 731 \\
\hline 39,40 &, 024 & 245 &, $200^{*}$ &, 996 & 245 &, 989 \\
\hline
\end{tabular}


X AXIS Tests of Normality

\begin{tabular}{|c|c|c|c|c|c|c|}
\hline & \multicolumn{3}{|c|}{ Kolmogorov-Smirnov ${ }^{a}$} & \multicolumn{3}{|c|}{ Shapiro-Wilk } \\
\hline & Statistic & $d f$ & Sig. & Statistic & $d f$ & Sig. \\
\hline 41,42 & ,026 & 245 &, $200^{*}$ & ,988 & 245 & ,037 \\
\hline 43,44 & ,053 & 245 & ,093 & ,991 & 245 & ,112 \\
\hline 45,46 & ,033 & 245 &, $200^{*}$ & ,989 & 245 & ,052 \\
\hline 47,48 & ,034 & 245 &, $200^{*}$ & ,987 & 245 &, 031 \\
\hline 49,50 & ,025 & 245 & ,200* & ,998 & 245 & ,993 \\
\hline 51,52 & ,033 & 245 & ,200* & ,995 & 245 & ,556 \\
\hline 53,54 & ,038 & 245 &, $200^{*}$ & ,990 & 245 & ,094 \\
\hline 55,56 & ,043 & 245 &, $200^{*}$ & ,996 & 245 & ,775 \\
\hline 57,58 & ,036 & 245 &, $200^{*}$ & ,991 & 245 & , 157 \\
\hline 59,60 & ,032 & 245 &, $200^{*}$ & ,994 & 245 & ,472 \\
\hline 61,62 &, 039 & 245 & ,200* & ,996 & 245 & ,785 \\
\hline 63,64 & ,030 & 245 & ,200* & ,996 & 245 & ,830 \\
\hline 65,66 & ,033 & 245 & ,200* & ,997 & 245 & ,921 \\
\hline 67,68 & ,035 & 245 &, $200^{*}$ & ,996 & 245 & ,764 \\
\hline 69,70 &, 040 & 245 &, $200^{*}$ & ,994 & 245 & ,494 \\
\hline 71,72 & ,047 & 245 &, $200^{*}$ & ,993 & 245 & ,321 \\
\hline 73,74 & ,045 & 245 & ,200* & ,993 & 245 & ,280 \\
\hline 75,76 & ,054 & 245 & ,081 & ,995 & 245 &, 581 \\
\hline 77,78 & ,058 & 245 & ,046 & ,992 & 245 & ,229 \\
\hline 79,80 & ,058 & 245 & ,046 & ,992 & 245 & ,229 \\
\hline 81,82 & ,044 & 245 &, $200^{*}$ & ,993 & 245 & ,252 \\
\hline 83,84 &, 064 & 245 & ,016 & ,993 & 245 & ,306 \\
\hline 85,86 & ,047 & 245 &, $200^{*}$ & ,994 & 245 & ,431 \\
\hline 87,88 &, 033 & 245 & ,200* & ,997 & 245 & ,888 \\
\hline 89,90 & ,028 & 245 &, $200^{*}$ & ,996 & 245 & ,753 \\
\hline 91,92 & ,032 & 245 & ,200* & ,996 & 245 & ,860 \\
\hline 93,94 &, 030 & 245 & ,200* & ,995 & 245 & ,627 \\
\hline 95,96 & ,028 & 245 &, $200^{*}$ & ,998 & 245 & ,985 \\
\hline 97,98 & ,038 & 245 &, $200^{*}$ & ,996 & 245 & ,833 \\
\hline 99,100 & ,038 & 245 & ,200* & ,996 & 245 & ,756 \\
\hline 101,102 &, 033 & 245 &, $200^{*}$ & ,997 & 245 & ,939 \\
\hline 103,104 & ,031 & 245 &, $200^{*}$ & ,997 & 245 & ,954 \\
\hline 105,106 &, 041 & 245 &, $200^{*}$ & ,996 & 245 & ,739 \\
\hline
\end{tabular}

*. This is a lower bound of the true significance.

a. Lilliefors Significance Correction 
Y AXIS Descriptives

\begin{tabular}{|c|c|c|c|c|}
\hline & & & Statistic & Std. Error \\
\hline \multirow[t]{13}{*}{11,12} & Mean & &,- 000244683 &, 0001247922 \\
\hline & \multirow{2}{*}{$\begin{array}{l}95 \% \text { Confidence Interval for } \\
\text { Mean }\end{array}$} & Lower Bound &,- 000490491 & \\
\hline & & Upper Bound & ,0000011244 & \\
\hline & $5 \%$ Trimmed Mean & &,- 000244534 & \\
\hline & Median & &,- 000304924 & \\
\hline & Variance & &, 000 & \\
\hline & Std. Deviation & & ,0019533073 & \\
\hline & Minimum & &,- 005624473 & \\
\hline & Maximum & & ,0059484379 & \\
\hline & Range & & 0115729113 & \\
\hline & Interquartile Range & & ,0028445576 & \\
\hline & Skewness & &, 053 & ,156 \\
\hline & Kurtosis & &,- 231 & ,310 \\
\hline \multirow[t]{13}{*}{13,14} & Mean & &,- 000295515 &, 0001245327 \\
\hline & \multirow{2}{*}{$\begin{array}{l}95 \% \text { Confidence Interval for } \\
\text { Mean }\end{array}$} & Lower Bound &,- 000540812 & \\
\hline & & Upper Bound &,- 000050219 & \\
\hline & $5 \%$ Trimmed Mean & &,- 000292888 & \\
\hline & Median & &,- 000327816 & \\
\hline & Variance & &, 000 & \\
\hline & Std. Deviation & & ,0019492453 & \\
\hline & Minimum & &,- 005314051 & \\
\hline & Maximum & & ,0056669056 & \\
\hline & Range & & ,0109809567 & \\
\hline & Interquartile Range & & ,0028267269 & \\
\hline & Skewness & &, 057 & ,156 \\
\hline & Kurtosis & &,- 315 & ,310 \\
\hline \multirow[t]{12}{*}{15,16} & Mean & &,- 000327807 & ,0001225270 \\
\hline & \multirow{2}{*}{$\begin{array}{l}95 \% \text { Confidence Interval for } \\
\text { Mean }\end{array}$} & Lower Bound &,- 000569152 & \\
\hline & & Upper Bound &,- 000086461 & \\
\hline & $5 \%$ Trimmed Mean & &,- 000330453 & \\
\hline & Median & &,- 000310285 & \\
\hline & Variance & &, 000 & \\
\hline & Std. Deviation & & ,0019178508 & \\
\hline & Minimum & &,- 005230925 & \\
\hline & Maximum & & ,0056001269 & \\
\hline & Range & & ,0108310518 & \\
\hline & Interquartile Range & & ,0026623411 & \\
\hline & Skewness & &, 066 & , 156 \\
\hline
\end{tabular}


Y AXIS Descriptives

\begin{tabular}{|c|c|c|c|c|}
\hline & & & Statistic & Std. Error \\
\hline & Kurtosis & &,- 316 & ,310 \\
\hline \multirow[t]{13}{*}{17,18} & Mean & &,- 000359580 & ,0001214608 \\
\hline & \multirow{2}{*}{$\begin{array}{l}95 \% \text { Confidence Interval for } \\
\text { Mean }\end{array}$} & Lower Bound &,- 000598825 & \\
\hline & & Upper Bound &,- 000120334 & \\
\hline & $5 \%$ Trimmed Mean & &,- 000368113 & \\
\hline & Median & &,- 000413554 & \\
\hline & Variance & &, 000 & \\
\hline & Std. Deviation & & ,0019011616 & \\
\hline & Minimum & &,- 004884456 & \\
\hline & Maximum & & ,0059847481 & \\
\hline & Range & & ,0108692037 & \\
\hline & Interquartile Range & & ,0026478201 & \\
\hline & Skewness & &, 117 & , 156 \\
\hline & Kurtosis & &,- 200 & ,310 \\
\hline \multirow[t]{13}{*}{19,20} & Mean & &,- 000334691 & ,0001214029 \\
\hline & \multirow{2}{*}{$\begin{array}{l}95 \% \text { Confidence Interval for } \\
\text { Mean }\end{array}$} & Lower Bound &,- 000573822 & \\
\hline & & Upper Bound &,- 000095559 & \\
\hline & $5 \%$ Trimmed Mean & &,- 000334626 & \\
\hline & Median & &,- 000354197 & \\
\hline & Variance & & ,000 & \\
\hline & Std. Deviation & & ,0019002560 & \\
\hline & Minimum & &,- 004848907 & \\
\hline & Maximum & & ,0056704343 & \\
\hline & Range & & 0105193410 & \\
\hline & Interquartile Range & & ,0026878314 & \\
\hline & Skewness & &, 057 & ,156 \\
\hline & Kurtosis & &,- 286 & ,310 \\
\hline \multirow[t]{11}{*}{21,22} & Mean & &,- 000284392 & ,0001214022 \\
\hline & \multirow{2}{*}{$\begin{array}{l}95 \% \text { Confidence Interval for } \\
\text { Mean }\end{array}$} & Lower Bound &,- 000523522 & \\
\hline & & Upper Bound &,- 000045262 & \\
\hline & $5 \%$ Trimmed Mean & &,- 000291795 & \\
\hline & Median & &,- 000330560 & \\
\hline & Variance & &, 000 & \\
\hline & Std. Deviation & & ,0019002448 & \\
\hline & Minimum & &,- 004484243 & \\
\hline & Maximum & & ,0060552953 & \\
\hline & Range & & 0105395387 & \\
\hline & Interquartile Range & & ,0027726032 & \\
\hline
\end{tabular}


Y AXIS Descriptives

\begin{tabular}{|c|c|c|c|c|}
\hline & & & Statistic & Std. Error \\
\hline & \multicolumn{2}{|l|}{ Skewness } &, 121 & ,156 \\
\hline & \multicolumn{2}{|l|}{ Kurtosis } &,- 259 & ,310 \\
\hline \multirow[t]{13}{*}{23,24} & \multicolumn{2}{|l|}{ Mean } &,- 000200313 & ,0001202109 \\
\hline & \multirow{2}{*}{$\begin{array}{l}95 \% \text { Confidence Interval for } \\
\text { Mean }\end{array}$} & Lower Bound &,- 000437096 & \\
\hline & & Upper Bound &, 0000364705 & \\
\hline & \multicolumn{2}{|l|}{$5 \%$ Trimmed Mean } &,- 000204088 & \\
\hline & \multicolumn{2}{|l|}{ Median } &,- 000229420 & \\
\hline & \multicolumn{2}{|l|}{ Variance } &, 000 & \\
\hline & \multicolumn{2}{|l|}{ Std. Deviation } & ,0018815982 & \\
\hline & \multicolumn{2}{|l|}{ Minimum } &,- 004811649 & \\
\hline & \multicolumn{2}{|l|}{ Maximum } &, 0057631615 & \\
\hline & \multicolumn{2}{|l|}{ Range } & ,0105748102 & \\
\hline & \multicolumn{2}{|l|}{ Interquartile Range } &, 0025733468 & \\
\hline & \multicolumn{2}{|l|}{ Skewness } & ,102 & ,156 \\
\hline & \multicolumn{2}{|l|}{ Kurtosis } &,- 189 &, 310 \\
\hline \multirow[t]{13}{*}{25,26} & \multicolumn{2}{|l|}{ Mean } &,- 000083471 & ,0001151190 \\
\hline & \multirow{2}{*}{$\begin{array}{l}95 \% \text { Confidence Interval for } \\
\text { Mean }\end{array}$} & Lower Bound &,- 000310224 & \\
\hline & & Upper Bound & ,0001432833 & \\
\hline & \multicolumn{2}{|l|}{$5 \%$ Trimmed Mean } &,- 000095461 & \\
\hline & \multicolumn{2}{|l|}{ Median } &,- 000147025 & \\
\hline & \multicolumn{2}{|l|}{ Variance } &, 000 & \\
\hline & \multicolumn{2}{|l|}{ Std. Deviation } &, 0018018970 & \\
\hline & \multicolumn{2}{|l|}{ Minimum } &,- 004314668 & \\
\hline & \multicolumn{2}{|l|}{ Maximum } &, 0052344175 & \\
\hline & \multicolumn{2}{|l|}{ Range } & ,0095490860 & \\
\hline & \multicolumn{2}{|l|}{ Interquartile Range } & ,0025607766 & \\
\hline & \multicolumn{2}{|l|}{ Skewness } &, 131 & ,156 \\
\hline & Kurtosis & &,- 287 & ,310 \\
\hline 27,28 & Mean & &, 0000161071 &, 0001060671 \\
\hline & $95 \%$ Confidence Interval for & Lower Bound &,- 000192817 & \\
\hline & Mean & Upper Bound &, 0002250311 & \\
\hline & $5 \%$ Trimmed Mean & &, 0000026116 & \\
\hline & Median & &, 0000766338 & \\
\hline & Variance & &, 000 & \\
\hline & Std. Deviation & &, 0016602125 & \\
\hline & Minimum & &,- 003964513 & \\
\hline & Maximum & &, 0051570260 & \\
\hline & Range & & ,0091215386 & \\
\hline
\end{tabular}


Y AXIS Descriptives

\begin{tabular}{|c|c|c|c|c|}
\hline & & & Statistic & Std. Error \\
\hline & \multicolumn{2}{|l|}{ Interquartile Range } & ,0022683252 & \\
\hline & \multicolumn{2}{|l|}{ Skewness } &, 145 & ,156 \\
\hline & \multicolumn{2}{|l|}{ Kurtosis } &,- 247 & ,310 \\
\hline \multirow[t]{13}{*}{29,30} & \multicolumn{2}{|l|}{ Mean } & ,0001288098 & ,0000931497 \\
\hline & \multirow{2}{*}{$\begin{array}{l}\text { 95\% Confidence Interval for } \\
\text { Mean }\end{array}$} & Lower Bound &,- 000054670 & \\
\hline & & Upper Bound & ,0003122900 & \\
\hline & \multicolumn{2}{|l|}{$5 \%$ Trimmed Mean } & ,0001141128 & \\
\hline & \multicolumn{2}{|l|}{ Median } & ,0001351496 & \\
\hline & \multicolumn{2}{|l|}{ Variance } & ,000 & \\
\hline & \multicolumn{2}{|l|}{ Std. Deviation } & ,0014580240 & \\
\hline & \multicolumn{2}{|l|}{ Minimum } &,- 003849334 & \\
\hline & \multicolumn{2}{|l|}{ Maximum } &, 0043585721 & \\
\hline & \multicolumn{2}{|l|}{ Range } & ,0082079064 & \\
\hline & \multicolumn{2}{|l|}{ Interquartile Range } & ,0019292265 & \\
\hline & \multicolumn{2}{|l|}{ Skewness } &, 167 & ,156 \\
\hline & \multicolumn{2}{|l|}{ Kurtosis } &,- 086 & ,310 \\
\hline \multirow[t]{13}{*}{31,32} & \multicolumn{2}{|l|}{ Mean } & ,0002022773 & ,0000788557 \\
\hline & \multirow{2}{*}{$\begin{array}{l}95 \% \text { Confidence Interval for } \\
\text { Mean }\end{array}$} & Lower Bound &, 0000469525 & \\
\hline & & Upper Bound & ,0003576022 & \\
\hline & \multicolumn{2}{|l|}{$5 \%$ Trimmed Mean } & ,0001910732 & \\
\hline & \multicolumn{2}{|l|}{ Median } & ,0002409878 & \\
\hline & \multicolumn{2}{|l|}{ Variance } & ,000 & \\
\hline & \multicolumn{2}{|l|}{ Std. Deviation } & ,0012342874 & \\
\hline & \multicolumn{2}{|l|}{ Minimum } &,- 002777159 & \\
\hline & \multicolumn{2}{|l|}{ Maximum } & ,0039002995 & \\
\hline & \multicolumn{2}{|l|}{ Range } & ,0066774582 & \\
\hline & \multicolumn{2}{|l|}{ Interquartile Range } & ,0015561870 & \\
\hline & Skewness & & ,152 & ,156 \\
\hline & Kurtosis & &,- 033 & ,310 \\
\hline 33,34 & Mean & & ,0002932439 &, 0000648629 \\
\hline & $95 \%$ Confidence Interval for & Lower Bound & ,0001654813 & \\
\hline & Mean & Upper Bound &, 0004210065 & \\
\hline & 5\% Trimmed Mean & & ,0002951889 & \\
\hline & Median & & ,0002799194 & \\
\hline & Variance & & ,000 & \\
\hline & Std. Deviation & & ,0010152645 & \\
\hline & Minimum & &,- 002228756 & \\
\hline & Maximum & & ,0034737345 & \\
\hline
\end{tabular}


Y AXIS Descriptives

\begin{tabular}{|c|c|c|c|c|}
\hline & & & Statistic & Std. Error \\
\hline & \multicolumn{2}{|l|}{ Range } & ,0057024907 & \\
\hline & \multicolumn{2}{|l|}{ Interquartile Range } & ,0012232188 & \\
\hline & \multicolumn{2}{|l|}{ Skewness } & 025 & ,156 \\
\hline & \multicolumn{2}{|l|}{ Kurtosis } & ,152 & ,310 \\
\hline \multirow[t]{13}{*}{35,36} & \multicolumn{2}{|l|}{ Mean } & ,0003036773 & ,0000560906 \\
\hline & \multirow{2}{*}{$\begin{array}{l}95 \% \text { Confidence Interval for } \\
\text { Mean }\end{array}$} & Lower Bound &, 0001931937 & \\
\hline & & Upper Bound &, 0004141609 & \\
\hline & \multicolumn{2}{|l|}{$5 \%$ Trimmed Mean } &, 0003096451 & \\
\hline & \multicolumn{2}{|l|}{ Median } &, 0003425266 & \\
\hline & \multicolumn{2}{|l|}{ Variance } &, 000 & \\
\hline & \multicolumn{2}{|l|}{ Std. Deviation } & ,0008779571 & \\
\hline & \multicolumn{2}{|l|}{ Minimum } &,- 001991866 & \\
\hline & \multicolumn{2}{|l|}{ Maximum } & ,0026422577 & \\
\hline & \multicolumn{2}{|l|}{ Range } & ,0046341238 & \\
\hline & \multicolumn{2}{|l|}{ Interquartile Range } &, 0011846316 & \\
\hline & \multicolumn{2}{|l|}{ Skewness } &,- 102 & ,156 \\
\hline & \multicolumn{2}{|l|}{ Kurtosis } &,- 250 & ,310 \\
\hline \multirow[t]{13}{*}{37,38} & \multicolumn{2}{|l|}{ Mean } &, 0003272164 &, 0000555361 \\
\hline & \multirow{2}{*}{$\begin{array}{l}95 \% \text { Confidence Interval for } \\
\text { Mean }\end{array}$} & Lower Bound &, 0002178251 & \\
\hline & & Upper Bound &, 0004366076 & \\
\hline & \multicolumn{2}{|l|}{$5 \%$ Trimmed Mean } & ,0003528120 & \\
\hline & \multicolumn{2}{|l|}{ Median } &, 0003671443 & \\
\hline & \multicolumn{2}{|l|}{ Variance } &, 000 & \\
\hline & \multicolumn{2}{|l|}{ Std. Deviation } & ,0008692769 & \\
\hline & \multicolumn{2}{|l|}{ Minimum } &,- 002176287 & \\
\hline & \multicolumn{2}{|l|}{ Maximum } & ,0021050400 & \\
\hline & \multicolumn{2}{|l|}{ Range } & ,0042813268 & \\
\hline & Interquartile Range & & ,0011584471 & \\
\hline & Skewness & &,- 397 & ,156 \\
\hline & Kurtosis & &,- 080 & ,310 \\
\hline 39,40 & Mean & &, 0003276207 &, 0000622190 \\
\hline & $95 \%$ Confidence Interval for & Lower Bound & ,0002050659 & \\
\hline & IVlean & Upper Bound &, 0004501756 & \\
\hline & $5 \%$ Trimmed Mean & & ,0003439565 & \\
\hline & Median & & ,0003994131 & \\
\hline & Variance & &, 000 & \\
\hline & Std. Deviation & & ,0009738810 & \\
\hline & Minimum & &,- 002487050 & \\
\hline
\end{tabular}


Y AXIS Descriptives

\begin{tabular}{|c|c|c|c|c|}
\hline & & & Statistic & Std. Error \\
\hline & \multicolumn{2}{|l|}{ Maximum } & ,0031571762 & \\
\hline & \multicolumn{2}{|l|}{ Range } & ,0056442261 & \\
\hline & \multicolumn{2}{|l|}{ Interquartile Range } & ,0013050076 & \\
\hline & \multicolumn{2}{|l|}{ Skewness } &,- 293 & ,156 \\
\hline & \multicolumn{2}{|l|}{ Kurtosis } &,- 030 & ,310 \\
\hline \multirow[t]{13}{*}{41,42} & \multicolumn{2}{|l|}{ Mean } &, 0003674284 & ,0000734537 \\
\hline & \multirow{2}{*}{$\begin{array}{l}95 \% \text { Confidence Interval for } \\
\text { Mean }\end{array}$} & Lower Bound & ,0002227441 & \\
\hline & & Upper Bound &, 0005121128 & \\
\hline & \multicolumn{2}{|l|}{$5 \%$ Trimmed Mean } & ,0003880302 & \\
\hline & \multicolumn{2}{|l|}{ Median } &, 0004368752 & \\
\hline & \multicolumn{2}{|l|}{ Variance } &, 000 & \\
\hline & \multicolumn{2}{|l|}{ Std. Deviation } & ,0011497330 & \\
\hline & \multicolumn{2}{|l|}{ Minimum } &,- 003291129 & \\
\hline & \multicolumn{2}{|l|}{ Maximum } & ,0043469943 & \\
\hline & \multicolumn{2}{|l|}{ Range } & ,0076381236 & \\
\hline & \multicolumn{2}{|l|}{ Interquartile Range } & ,0014772339 & \\
\hline & \multicolumn{2}{|l|}{ Skewness } &,- 203 & ,156 \\
\hline & \multicolumn{2}{|l|}{ Kurtosis } & ,246 & ,310 \\
\hline \multirow[t]{13}{*}{43,44} & \multicolumn{2}{|l|}{ Mean } &,- 000237104 & ,0000807752 \\
\hline & \multirow{2}{*}{$\begin{array}{l}95 \% \text { Confidence Interval for } \\
\text { Mean }\end{array}$} & Lower Bound &,- 000396210 & \\
\hline & & Upper Bound &,- 000077999 & \\
\hline & \multicolumn{2}{|l|}{$5 \%$ Trimmed Mean } &,- 000233175 & \\
\hline & \multicolumn{2}{|l|}{ Median } &,- 000246702 & \\
\hline & \multicolumn{2}{|l|}{ Variance } &, 000 & \\
\hline & \multicolumn{2}{|l|}{ Std. Deviation } & ,0012643326 & \\
\hline & \multicolumn{2}{|l|}{ Minimum } &,- 004529149 & \\
\hline & \multicolumn{2}{|l|}{ Maximum } &, 0037124977 & \\
\hline & Range & & ,0082416470 & \\
\hline & Interquartile Range & & ,0017211066 & \\
\hline & Skewness & &,- 034 & ,156 \\
\hline & Kurtosis & & ,387 & ,310 \\
\hline 45,46 & Mean & &,- 000185294 & ,0000777172 \\
\hline & $95 \%$ Confidence Interval for & Lower Bound &,- 000338376 & \\
\hline & Mean & Upper Bound &,- 000032212 & \\
\hline & $5 \%$ Trimmed Mean & &,- 000185410 & \\
\hline & Median & &,- 000250746 & \\
\hline & Variance & &, 000 & \\
\hline & Std. Deviation & &, 0012164666 & \\
\hline
\end{tabular}


Y AXIS Descriptives

\begin{tabular}{|c|c|c|c|c|}
\hline & & & Statistic & Std. Error \\
\hline & \multicolumn{2}{|l|}{ Minimum } &,- 003981504 & \\
\hline & \multicolumn{2}{|l|}{ Maximum } & ,0041390910 & \\
\hline & \multicolumn{2}{|l|}{ Range } & ,0081205951 & \\
\hline & \multicolumn{2}{|l|}{ Interquartile Range } & ,0015075906 & \\
\hline & \multicolumn{2}{|l|}{ Skewness } &, 063 & , 156 \\
\hline & \multicolumn{2}{|l|}{ Kurtosis } & ,971 & ,310 \\
\hline \multirow[t]{13}{*}{47,48} & \multicolumn{2}{|l|}{ Mean } &,- 000259171 & ,0000773953 \\
\hline & \multirow{2}{*}{$\begin{array}{l}95 \% \text { Confidence Interval for } \\
\text { Mean }\end{array}$} & Lower Bound &,- 000411619 & \\
\hline & & Upper Bound &,- 000106723 & \\
\hline & \multicolumn{2}{|l|}{ 5\% Trimmed Mean } &,- 000264843 & \\
\hline & \multicolumn{2}{|l|}{ Median } &,- 000267945 & \\
\hline & \multicolumn{2}{|l|}{ Variance } &, 000 & \\
\hline & \multicolumn{2}{|l|}{ Std. Deviation } & ,0012114281 & \\
\hline & \multicolumn{2}{|l|}{ Minimum } &,- 003984324 & \\
\hline & \multicolumn{2}{|l|}{ Maximum } & ,0031303196 & \\
\hline & \multicolumn{2}{|l|}{ Range } & ,0071146431 & \\
\hline & \multicolumn{2}{|l|}{ Interquartile Range } & ,0016129389 & \\
\hline & \multicolumn{2}{|l|}{ Skewness } &, 060 & , 156 \\
\hline & \multicolumn{2}{|l|}{ Kurtosis } &, 023 & ,310 \\
\hline \multirow[t]{13}{*}{49,50} & \multicolumn{2}{|l|}{ Mean } &,- 000304502 & ,0000863651 \\
\hline & \multirow{2}{*}{$\begin{array}{l}95 \% \text { Confidence Interval for } \\
\text { Mean }\end{array}$} & Lower Bound &,- 000474619 & \\
\hline & & Upper Bound &,- 000134386 & \\
\hline & \multicolumn{2}{|l|}{$5 \%$ Trimmed Mean } &,- 000311776 & \\
\hline & \multicolumn{2}{|l|}{ Median } &,- 000303258 & \\
\hline & \multicolumn{2}{|l|}{ Variance } &, 000 & \\
\hline & \multicolumn{2}{|l|}{ Std. Deviation } & ,0013518279 & \\
\hline & \multicolumn{2}{|l|}{ Minimum } &,- 004204592 & \\
\hline & Maximum & & ,0033179714 & \\
\hline & Range & & ,0075225633 & \\
\hline & Interquartile Range & & ,0020692090 & \\
\hline & Skewness & &,- 017 & , 156 \\
\hline & Kurtosis & &,- 309 & ,310 \\
\hline 51,52 & Mean & &,- 000166892 & ,0000992228 \\
\hline & $95 \%$ Confidence Interval for & Lower Bound &,- 000362334 & \\
\hline & IVean & Upper Bound & ,0000285506 & \\
\hline & $5 \%$ Trimmed Mean & &,- 000173390 & \\
\hline & Median & &,- 000221043 & \\
\hline & Variance & &, 000 & \\
\hline
\end{tabular}


Y AXIS Descriptives

\begin{tabular}{|c|c|c|c|c|}
\hline & & & Statistic & Std. Error \\
\hline & \multicolumn{2}{|l|}{ Std. Deviation } & ,0015530824 & \\
\hline & \multicolumn{2}{|l|}{ Minimum } &,- 004170230 & \\
\hline & \multicolumn{2}{|l|}{ Maximum } & ,0040470415 & \\
\hline & \multicolumn{2}{|l|}{ Range } & ,0082172719 & \\
\hline & \multicolumn{2}{|l|}{ Interquartile Range } & ,0022172354 & \\
\hline & \multicolumn{2}{|l|}{ Skewness } &,- 003 & ,156 \\
\hline & \multicolumn{2}{|l|}{ Kurtosis } &,- 222 & ,310 \\
\hline \multirow[t]{13}{*}{53,54} & \multicolumn{2}{|l|}{ Mean } &,- 000083900 & ,0000971946 \\
\hline & \multirow{2}{*}{$\begin{array}{l}\text { 95\% Confidence Interval for } \\
\text { Mean }\end{array}$} & Lower Bound &,- 000275348 & \\
\hline & & Upper Bound & ,0001075473 & \\
\hline & \multicolumn{2}{|l|}{$5 \%$ Trimmed Mean } &,- 000086800 & \\
\hline & \multicolumn{2}{|l|}{ Median } &,- 000140053 & \\
\hline & \multicolumn{2}{|l|}{ Variance } &, 000 & \\
\hline & \multicolumn{2}{|l|}{ Std. Deviation } &, 0015213364 & \\
\hline & \multicolumn{2}{|l|}{ Minimum } &,- 003865411 & \\
\hline & \multicolumn{2}{|l|}{ Maximum } & ,0037977417 & \\
\hline & \multicolumn{2}{|l|}{ Range } & ,0076631528 & \\
\hline & \multicolumn{2}{|l|}{ Interquartile Range } & ,0021808381 & \\
\hline & \multicolumn{2}{|l|}{ Skewness } &, 025 & ,156 \\
\hline & \multicolumn{2}{|l|}{ Kurtosis } &,- 256 & ,310 \\
\hline \multirow[t]{13}{*}{55,56} & \multicolumn{2}{|l|}{ Mean } &,- 000189767 &, 0000821544 \\
\hline & \multirow{2}{*}{$\begin{array}{l}\text { 95\% Confidence Interval for } \\
\text { Mean }\end{array}$} & Lower Bound &,- 000351589 & \\
\hline & & Upper Bound &,- 000027944 & \\
\hline & \multicolumn{2}{|l|}{$5 \%$ Trimmed Mean } &,- 000201396 & \\
\hline & \multicolumn{2}{|l|}{ Median } &,- 000185942 & \\
\hline & \multicolumn{2}{|l|}{ Variance } &, 000 & \\
\hline & \multicolumn{2}{|l|}{ Std. Deviation } & ,0012859205 & \\
\hline & Minimum & &,- 004109148 & \\
\hline & Maximum & & ,0032802342 & \\
\hline & Range & & ,0073893817 & \\
\hline & Interquartile Range & & ,0018261882 & \\
\hline & Skewness & &, 089 & , 156 \\
\hline & Kurtosis & &,- 184 & ,310 \\
\hline 57,58 & Mean & &,- 000195194 & ,0000721995 \\
\hline & $95 \%$ Confidence Interval for & Lower Bound &,- 000337408 & \\
\hline & IVlean & Upper Bound &,- 000052981 & \\
\hline & $5 \%$ Trimmed Mean & &,- 000215092 & \\
\hline & Median & &,- 000286916 & \\
\hline
\end{tabular}


Y AXIS Descriptives

\begin{tabular}{|c|c|c|c|c|}
\hline & & & Statistic & Std. Error \\
\hline & \multicolumn{2}{|l|}{ Variance } &, 000 & \\
\hline & \multicolumn{2}{|l|}{ Std. Deviation } & ,0011301003 & \\
\hline & \multicolumn{2}{|l|}{ Minimum } &,- 003428758 & \\
\hline & \multicolumn{2}{|l|}{ Maximum } & ,0033714833 & \\
\hline & \multicolumn{2}{|l|}{ Range } & ,0068002415 & \\
\hline & \multicolumn{2}{|l|}{ Interquartile Range } & ,0014769673 & \\
\hline & \multicolumn{2}{|l|}{ Skewness } & ,225 & , 156 \\
\hline & \multicolumn{2}{|l|}{ Kurtosis } & 208 & ,310 \\
\hline \multirow[t]{13}{*}{59,60} & \multicolumn{2}{|l|}{ Mean } &,- 000246237 &, 0000691215 \\
\hline & \multirow{2}{*}{$\begin{array}{l}95 \% \text { Confidence Interval for } \\
\text { Mean }\end{array}$} & Lower Bound &,- 000382388 & \\
\hline & & Upper Bound &,- 000110086 & \\
\hline & \multicolumn{2}{|l|}{$5 \%$ Trimmed Mean } &,- 000251021 & \\
\hline & \multicolumn{2}{|l|}{ Median } &,- 000268283 & \\
\hline & \multicolumn{2}{|l|}{ Variance } &, 000 & \\
\hline & \multicolumn{2}{|l|}{ Std. Deviation } & ,0010819226 & \\
\hline & \multicolumn{2}{|l|}{ Minimum } &,- 003677678 & \\
\hline & \multicolumn{2}{|l|}{ Maximum } & ,0034019818 & \\
\hline & \multicolumn{2}{|l|}{ Range } & ,0070796594 & \\
\hline & \multicolumn{2}{|l|}{ Interquartile Range } & 0013419178 & \\
\hline & \multicolumn{2}{|l|}{ Skewness } &, 069 & , 156 \\
\hline & \multicolumn{2}{|l|}{ Kurtosis } &, 546 & ,310 \\
\hline \multirow[t]{13}{*}{61,62} & \multicolumn{2}{|l|}{ Mean } &, 0001321100 &, 0000553245 \\
\hline & \multirow{2}{*}{$\begin{array}{l}95 \% \text { Confidence Interval for } \\
\text { Mean }\end{array}$} & Lower Bound & ,0000231354 & \\
\hline & & Upper Bound &, 0002410846 & \\
\hline & \multicolumn{2}{|l|}{$5 \%$ Trimmed Mean } &, 0001306056 & \\
\hline & \multicolumn{2}{|l|}{ Median } & ,0000686083 & \\
\hline & \multicolumn{2}{|l|}{ Variance } &, 000 & \\
\hline & Std. Deviation & & ,0008659658 & \\
\hline & Minimum & &,- 002846134 & \\
\hline & Maximum & & ,0022856627 & \\
\hline & Range & &, 0051317966 & \\
\hline & Interquartile Range & & ,0012453910 & \\
\hline & Skewness & &, 007 & , 156 \\
\hline & Kurtosis & & ,110 & ,310 \\
\hline 63,64 & Mean & & ,0001428781 &, 0000524419 \\
\hline & $95 \%$ Confidence Interval for & Lower Bound &, 0000395815 & \\
\hline & & Upper Bound & ,0002461748 & \\
\hline & $5 \%$ Trimmed Mean & & ,0001488662 & \\
\hline
\end{tabular}


Y AXIS Descriptives

\begin{tabular}{|c|c|c|c|c|}
\hline & & & Statistic & Std. Error \\
\hline & \multicolumn{2}{|l|}{ Median } & ,0002011775 & \\
\hline & \multicolumn{2}{|l|}{ Variance } &, 000 & \\
\hline & \multicolumn{2}{|l|}{ Std. Deviation } & ,0008208459 & \\
\hline & \multicolumn{2}{|l|}{ Minimum } &,- 002778163 & \\
\hline & \multicolumn{2}{|l|}{ Maximum } & ,0020327061 & \\
\hline & \multicolumn{2}{|l|}{ Range } & ,0048108695 & \\
\hline & \multicolumn{2}{|l|}{ Interquartile Range } & ,0012640085 & \\
\hline & \multicolumn{2}{|l|}{ Skewness } &,- 168 & ,156 \\
\hline & \multicolumn{2}{|l|}{ Kurtosis } &,- 032 & ,310 \\
\hline \multirow[t]{13}{*}{65,66} & \multicolumn{2}{|l|}{ Mean } &, 0002019674 & ,0000495643 \\
\hline & \multirow{2}{*}{$\begin{array}{l}95 \% \text { Confidence Interval for } \\
\text { Mean }\end{array}$} & Lower Bound &, 0001043388 & \\
\hline & & Upper Bound & ,0002995959 & \\
\hline & \multicolumn{2}{|l|}{$5 \%$ Trimmed Mean } & ,0002117574 & \\
\hline & \multicolumn{2}{|l|}{ Median } &, 0002675326 & \\
\hline & \multicolumn{2}{|l|}{ Variance } &, 000 & \\
\hline & \multicolumn{2}{|l|}{ Std. Deviation } & ,0007758045 & \\
\hline & \multicolumn{2}{|l|}{ Minimum } &,- 002391422 & \\
\hline & \multicolumn{2}{|l|}{ Maximum } &, 0020628619 & \\
\hline & \multicolumn{2}{|l|}{ Range } & ,0044542842 & \\
\hline & \multicolumn{2}{|l|}{ Interquartile Range } &, 0011296647 & \\
\hline & \multicolumn{2}{|l|}{ Skewness } &,- 173 & ,156 \\
\hline & \multicolumn{2}{|l|}{ Kurtosis } &,- 041 & ,310 \\
\hline \multirow[t]{13}{*}{67,68} & \multicolumn{2}{|l|}{ Mean } & ,0001854673 & ,0000494553 \\
\hline & \multirow{2}{*}{$\begin{array}{l}95 \% \text { Confidence Interval for } \\
\text { Mean }\end{array}$} & Lower Bound & ,0000880535 & \\
\hline & & Upper Bound & ,0002828812 & \\
\hline & \multicolumn{2}{|l|}{$5 \%$ Trimmed Mean } & ,0001882861 & \\
\hline & \multicolumn{2}{|l|}{ Median } & ,0002099886 & \\
\hline & Variance & &, 000 & \\
\hline & Std. Deviation & & ,0007740984 & \\
\hline & Minimum & &,- 001930642 & \\
\hline & Maximum & & ,0022723149 & \\
\hline & Range & &, 0042029564 & \\
\hline & Interquartile Range & & ,0011576561 & \\
\hline & Skewness & &,- 020 & ,156 \\
\hline & Kurtosis & &,- 395 & ,310 \\
\hline 69,70 & Mean & &, 0000238691 & ,0000530022 \\
\hline & $95 \%$ Confidence Interval for & Lower Bound &,- 000080531 & \\
\hline & IVlean & Upper Bound & ,0001282694 & \\
\hline
\end{tabular}


Y AXIS Descriptives

\begin{tabular}{|c|c|c|c|c|}
\hline & & & Statistic & Std. Error \\
\hline & \multicolumn{2}{|l|}{$5 \%$ Trimmed Mean } & ,0000219066 & \\
\hline & \multicolumn{2}{|l|}{ Median } & ,0000497583 & \\
\hline & \multicolumn{2}{|l|}{ Variance } &, 000 & \\
\hline & \multicolumn{2}{|l|}{ Std. Deviation } & ,0008296155 & \\
\hline & \multicolumn{2}{|l|}{ Minimum } &,- 001769340 & \\
\hline & \multicolumn{2}{|l|}{ Maximum } & ,0023747690 & \\
\hline & \multicolumn{2}{|l|}{ Range } & ,0041441089 & \\
\hline & \multicolumn{2}{|l|}{ Interquartile Range } & ,0011429362 & \\
\hline & \multicolumn{2}{|l|}{ Skewness } & ,057 & ,156 \\
\hline & \multicolumn{2}{|l|}{ Kurtosis } &,- 410 & ,310 \\
\hline \multirow[t]{13}{*}{71,72} & \multicolumn{2}{|l|}{ Mean } & ,0000967947 & ,0000515778 \\
\hline & \multirow{2}{*}{$\begin{array}{l}95 \% \text { Confidence Interval for } \\
\text { Mean }\end{array}$} & Lower Bound &,- 000004800 & \\
\hline & & Upper Bound & ,0001983892 & \\
\hline & \multicolumn{2}{|l|}{$5 \%$ Trimmed Mean } & ,0000995833 & \\
\hline & \multicolumn{2}{|l|}{ Median } &, 0001453698 & \\
\hline & \multicolumn{2}{|l|}{ Variance } &, 000 & \\
\hline & \multicolumn{2}{|l|}{ Std. Deviation } & ,0008073204 & \\
\hline & \multicolumn{2}{|l|}{ Minimum } &,- 002180114 & \\
\hline & \multicolumn{2}{|l|}{ Maximum } & ,0024696532 & \\
\hline & \multicolumn{2}{|l|}{ Range } & ,0046497676 & \\
\hline & \multicolumn{2}{|l|}{ Interquartile Range } &, 0011866402 & \\
\hline & \multicolumn{2}{|l|}{ Skewness } &,- 040 & ,156 \\
\hline & \multicolumn{2}{|l|}{ Kurtosis } &,- 318 & ,310 \\
\hline \multirow[t]{13}{*}{73,74} & \multicolumn{2}{|l|}{ Mean } & ,0001845866 &, 0000534973 \\
\hline & \multirow{2}{*}{$\begin{array}{l}95 \% \text { Confidence Interval for } \\
\text { Mean }\end{array}$} & Lower Bound & ,0000792112 & \\
\hline & & Upper Bound & ,0002899620 & \\
\hline & \multicolumn{2}{|l|}{$5 \%$ Trimmed Mean } & ,0001889585 & \\
\hline & \multicolumn{2}{|l|}{ Median } & ,0002396267 & \\
\hline & Variance & &, 000 & \\
\hline & Std. Deviation & & ,0008373649 & \\
\hline & Minimum & &,- 002514079 & \\
\hline & Maximum & & ,0023404309 & \\
\hline & Range & & ,0048545096 & \\
\hline & Interquartile Range & & ,0012808151 & \\
\hline & Skewness & &,- 117 & ,156 \\
\hline & Kurtosis & &,- 052 & ,310 \\
\hline 75,76 & Mean & & ,0002182225 & ,0000559053 \\
\hline
\end{tabular}


Y AXIS Descriptives

\begin{tabular}{|c|c|c|c|c|}
\hline & & & Statistic & Std. Error \\
\hline & \multirow{2}{*}{$\begin{array}{l}95 \% \text { Confidence Interval for } \\
\text { Mean }\end{array}$} & Lower Bound & ,0001081038 & \\
\hline & & Upper Bound &, 0003283411 & \\
\hline & $5 \%$ Trimmed Mean & & ,0002210880 & \\
\hline & Median & & ,0001915000 & \\
\hline & Variance & &, 000 & \\
\hline & Std. Deviation & &, 0008750570 & \\
\hline & Minimum & &,- 002815487 & \\
\hline & Maximum & & ,0023469415 & \\
\hline & Range & & ,0051624287 & \\
\hline & Interquartile Range & & ,0012291942 & \\
\hline & Skewness & &,- 113 & ,156 \\
\hline & Kurtosis & & ,218 & ,310 \\
\hline \multirow[t]{13}{*}{77,78} & Mean & & ,0004086501 & ,0000597536 \\
\hline & \multirow{2}{*}{$\begin{array}{l}95 \% \text { Confidence Interval for } \\
\text { Mean }\end{array}$} & Lower Bound & ,0002909514 & \\
\hline & & Upper Bound & ,0005263488 & \\
\hline & $5 \%$ Trimmed Mean & & ,0004066803 & \\
\hline & Median & &, 0003806149 & \\
\hline & Variance & &, 000 & \\
\hline & Std. Deviation & & ,0009352915 & \\
\hline & Minimum & &,- 002775008 & \\
\hline & Maximum & & ,0031084853 & \\
\hline & Range & & ,0058834930 & \\
\hline & Interquartile Range & & ,0012318402 & \\
\hline & Skewness & &, 043 & ,156 \\
\hline & Kurtosis & & ,250 & ,310 \\
\hline \multirow[t]{13}{*}{79,80} & Mean & & ,0004086501 & ,0000597536 \\
\hline & \multirow{2}{*}{$\begin{array}{l}95 \% \text { Confidence Interval for } \\
\text { Mean }\end{array}$} & Lower Bound & ,0002909514 & \\
\hline & & Upper Bound & ,0005263488 & \\
\hline & $5 \%$ Trimmed Mean & & ,0004066803 & \\
\hline & Median & & ,0003806149 & \\
\hline & Variance & &, 000 & \\
\hline & Std. Deviation & & ,0009352915 & \\
\hline & Minimum & &,- 002775008 & \\
\hline & Maximum & &, 0031084853 & \\
\hline & Range & & ,0058834930 & \\
\hline & Interquartile Range & & ,0012318402 & \\
\hline & Skewness & &, 043 & ,156 \\
\hline & Kurtosis & & ,250 & ,310 \\
\hline
\end{tabular}


Y AXIS Descriptives

\begin{tabular}{|c|c|c|c|c|}
\hline & & & Statistic & Std. Error \\
\hline \multirow[t]{13}{*}{81,82} & Mean & &,- 000190197 &, 0000646047 \\
\hline & \multirow{2}{*}{$\begin{array}{l}95 \% \text { Confidence Interval for } \\
\text { Mean }\end{array}$} & Lower Bound &,- 000317451 & \\
\hline & & Upper Bound &,- 000062943 & \\
\hline & $5 \%$ Trimmed Mean & &,- 000182528 & \\
\hline & Median & &,- 000202491 & \\
\hline & Variance & &, 000 & \\
\hline & Std. Deviation & & ,0010112228 & \\
\hline & Minimum & &,- 003099563 & \\
\hline & Maximum & & ,0022167714 & \\
\hline & Range & & ,0053163348 & \\
\hline & Interquartile Range & & ,0013892032 & \\
\hline & Skewness & &,- 169 & ,156 \\
\hline & Kurtosis & &,- 138 & ,310 \\
\hline \multirow[t]{13}{*}{83,84} & Mean & & ,0001946568 &, 0000760509 \\
\hline & \multirow{2}{*}{$\begin{array}{l}95 \% \text { Confidence Interval for } \\
\text { Mean }\end{array}$} & Lower Bound & ,0000448568 & \\
\hline & & Upper Bound & ,0003444568 & \\
\hline & $5 \%$ Trimmed Mean & & ,0001979172 & \\
\hline & Median & &, 0002154575 & \\
\hline & Variance & &, 000 & \\
\hline & Std. Deviation & & ,0011903844 & \\
\hline & Minimum & &,- 003013756 & \\
\hline & Maximum & & ,0032774352 & \\
\hline & Range & & ,0062911916 & \\
\hline & Interquartile Range & & ,0017002809 & \\
\hline & Skewness & &,- 038 & ,156 \\
\hline & Kurtosis & &,- 171 & ,310 \\
\hline \multirow[t]{12}{*}{85,86} & Mean & &, 0003148154 & ,0000730350 \\
\hline & \multirow{2}{*}{$\begin{array}{l}95 \% \text { Confidence Interval for } \\
\text { Mean }\end{array}$} & Lower Bound & ,0001709559 & \\
\hline & & Upper Bound & ,0004586749 & \\
\hline & $5 \%$ Trimmed Mean & & ,0003162045 & \\
\hline & Median & &, 0002602640 & \\
\hline & Variance & &, 000 & \\
\hline & Std. Deviation & & ,0011431784 & \\
\hline & Minimum & &,- 002717250 & \\
\hline & Maximum & & ,0036521427 & \\
\hline & Range & & ,0063693927 & \\
\hline & Interquartile Range & & ,0016627308 & \\
\hline & Skewness & &,- 042 & ,156 \\
\hline
\end{tabular}


Y AXIS Descriptives

\begin{tabular}{|c|c|c|c|c|}
\hline & & & Statistic & Std. Error \\
\hline & Kurtosis & &,- 125 & ,310 \\
\hline \multirow[t]{13}{*}{87,88} & Mean & & ,0002925784 & ,0000850266 \\
\hline & \multirow{2}{*}{$\begin{array}{l}95 \% \text { Confidence Interval for } \\
\text { Mean }\end{array}$} & Lower Bound & ,0001250987 & \\
\hline & & Upper Bound & ,0004600582 & \\
\hline & $5 \%$ Trimmed Mean & & ,0002943075 & \\
\hline & Median & & ,0002701815 & \\
\hline & Variance & &, 000 & \\
\hline & Std. Deviation & & ,0013308764 & \\
\hline & Minimum & &,- 003151281 & \\
\hline & Maximum & & ,0040445392 & \\
\hline & Range & & ,0071958198 & \\
\hline & Interquartile Range & & ,0020444959 & \\
\hline & Skewness & &,- 014 & , 156 \\
\hline & Kurtosis & &,- 373 & ,310 \\
\hline \multirow[t]{13}{*}{89,90} & Mean & & ,0002815765 & ,0001001301 \\
\hline & \multirow{2}{*}{$\begin{array}{l}95 \% \text { Confidence Interval for } \\
\text { Mean }\end{array}$} & Lower Bound & ,0000843468 & \\
\hline & & Upper Bound & ,0004788061 & \\
\hline & $5 \%$ Trimmed Mean & & ,0002811619 & \\
\hline & Median & & ,0003367688 & \\
\hline & Variance & &, 000 & \\
\hline & Std. Deviation & & ,0015672840 & \\
\hline & Minimum & &,- 003549944 & \\
\hline & Maximum & & ,0043712062 & \\
\hline & Range & & ,0079211497 & \\
\hline & Interquartile Range & & ,0022294183 & \\
\hline & Skewness & &,- 034 & , 156 \\
\hline & Kurtosis & &,- 420 & ,310 \\
\hline \multirow[t]{11}{*}{91,92} & Mean & &, 0002322817 & ,0000875840 \\
\hline & \multirow{2}{*}{$\begin{array}{l}95 \% \text { Confidence Interval for } \\
\text { Mean }\end{array}$} & Lower Bound & ,0000597645 & \\
\hline & & Upper Bound & ,0004047989 & \\
\hline & $5 \%$ Trimmed Mean & & ,0002282731 & \\
\hline & Median & & ,0001142037 & \\
\hline & Variance & &, 000 & \\
\hline & Std. Deviation & & ,0013709063 & \\
\hline & Minimum & &,- 003990093 & \\
\hline & Maximum & & ,0042777362 & \\
\hline & Range & & ,0082678292 & \\
\hline & Interquartile Range & & ,0018629280 & \\
\hline
\end{tabular}


Y AXIS Descriptives

\begin{tabular}{|c|c|c|c|c|}
\hline & & & Statistic & Std. Error \\
\hline & \multicolumn{2}{|l|}{ Skewness } &, 046 & , 156 \\
\hline & \multicolumn{2}{|l|}{ Kurtosis } &, 096 & ,310 \\
\hline \multirow[t]{13}{*}{93,94} & \multicolumn{2}{|l|}{ Mean } &,- 000054863 &, 0000780323 \\
\hline & \multirow{2}{*}{$\begin{array}{l}95 \% \text { Confidence Interval for } \\
\text { Mean }\end{array}$} & Lower Bound &,- 000208566 & \\
\hline & & Upper Bound & ,0000988399 & \\
\hline & \multicolumn{2}{|l|}{$5 \%$ Trimmed Mean } &,- 000071438 & \\
\hline & \multicolumn{2}{|l|}{ Median } &,- 000131402 & \\
\hline & \multicolumn{2}{|l|}{ Variance } &, 000 & \\
\hline & \multicolumn{2}{|l|}{ Std. Deviation } & ,0012213986 & \\
\hline & \multicolumn{2}{|l|}{ Minimum } &,- 004164715 & \\
\hline & \multicolumn{2}{|l|}{ Maximum } & ,0032298788 & \\
\hline & \multicolumn{2}{|l|}{ Range } & ,0073945936 & \\
\hline & \multicolumn{2}{|l|}{ Interquartile Range } & ,0017297109 & \\
\hline & \multicolumn{2}{|l|}{ Skewness } &, 094 & , 156 \\
\hline & \multicolumn{2}{|l|}{ Kurtosis } & ,371 & ,310 \\
\hline \multirow[t]{13}{*}{95,96} & \multicolumn{2}{|l|}{ Mean } &, 0001884256 & ,0000802886 \\
\hline & \multirow{2}{*}{$\begin{array}{l}95 \% \text { Confidence Interval for } \\
\text { Mean }\end{array}$} & Lower Bound & ,0000302783 & \\
\hline & & Upper Bound & ,0003465728 & \\
\hline & \multicolumn{2}{|l|}{$5 \%$ Trimmed Mean } &, 0001862152 & \\
\hline & \multicolumn{2}{|l|}{ Median } & ,0001098661 & \\
\hline & \multicolumn{2}{|l|}{ Variance } &, 000 & \\
\hline & \multicolumn{2}{|l|}{ Std. Deviation } & ,0012567156 & \\
\hline & \multicolumn{2}{|l|}{ Minimum } &,- 004189587 & \\
\hline & \multicolumn{2}{|l|}{ Maximum } & ,0038227344 & \\
\hline & \multicolumn{2}{|l|}{ Range } & ,0080123219 & \\
\hline & \multicolumn{2}{|l|}{ Interquartile Range } & ,0017045967 & \\
\hline & \multicolumn{2}{|l|}{ Skewness } &,- 012 & ,156 \\
\hline & Kurtosis & & ,378 & ,310 \\
\hline 97,98 & Mean & &,- 000096210 &, 0000987215 \\
\hline & $95 \%$ Confidence Interval for & Lower Bound &,- 000290666 & \\
\hline & Mean & Upper Bound & ,0000982447 & \\
\hline & $5 \%$ Trimmed Mean & &,- 000099723 & \\
\hline & Median & &,- 000135114 & \\
\hline & Variance & &, 000 & \\
\hline & Std. Deviation & & ,0015452364 & \\
\hline & Minimum & &,- 004349089 & \\
\hline & Maximum & & ,0037616077 & \\
\hline & Range & & 0081106971 & \\
\hline
\end{tabular}


Y AXIS Descriptives

\begin{tabular}{|c|c|c|c|c|}
\hline & & & Statistic & Std. Error \\
\hline & \multicolumn{2}{|l|}{ Interquartile Range } & ,0021809197 & \\
\hline & \multicolumn{2}{|l|}{ Skewness } &, 053 & ,156 \\
\hline & \multicolumn{2}{|l|}{ Kurtosis } &,- 206 & ,310 \\
\hline \multirow[t]{13}{*}{99,100} & \multicolumn{2}{|l|}{ Mean } &,- 000079174 & ,0000766426 \\
\hline & \multirow{2}{*}{$\begin{array}{l}\text { 95\% Confidence Interval for } \\
\text { Mean }\end{array}$} & Lower Bound &,- 000230140 & \\
\hline & & Upper Bound & ,0000717915 & \\
\hline & \multicolumn{2}{|l|}{$5 \%$ Trimmed Mean } &,- 000086544 & \\
\hline & \multicolumn{2}{|l|}{ Median } &,- 000125995 & \\
\hline & \multicolumn{2}{|l|}{ Variance } &, 000 & \\
\hline & \multicolumn{2}{|l|}{ Std. Deviation } & ,0011996464 & \\
\hline & \multicolumn{2}{|l|}{ Minimum } &,- 004777641 & \\
\hline & \multicolumn{2}{|l|}{ Maximum } & ,0031041802 & \\
\hline & \multicolumn{2}{|l|}{ Range } & ,0078818211 & \\
\hline & \multicolumn{2}{|l|}{ Interquartile Range } & ,0015197809 & \\
\hline & \multicolumn{2}{|l|}{ Skewness } &,- 046 & ,156 \\
\hline & \multicolumn{2}{|l|}{ Kurtosis } &, 764 & ,310 \\
\hline \multirow[t]{13}{*}{101,102} & \multicolumn{2}{|l|}{ Mean } &,- 000096588 & ,0000790601 \\
\hline & \multirow{2}{*}{$\begin{array}{l}95 \% \text { Confidence Interval for } \\
\text { Mean }\end{array}$} & Lower Bound &,- 000252315 & \\
\hline & & Upper Bound &, 0000591397 & \\
\hline & \multicolumn{2}{|l|}{$5 \%$ Trimmed Mean } &,- 000099303 & \\
\hline & \multicolumn{2}{|l|}{ Median } &,- 000136496 & \\
\hline & \multicolumn{2}{|l|}{ Variance } &, 000 & \\
\hline & \multicolumn{2}{|l|}{ Std. Deviation } &, 0012374867 & \\
\hline & \multicolumn{2}{|l|}{ Minimum } &,- 004611117 & \\
\hline & \multicolumn{2}{|l|}{ Maximum } & ,0034179833 & \\
\hline & \multicolumn{2}{|l|}{ Range } & ,0080291005 & \\
\hline & \multicolumn{2}{|l|}{ Interquartile Range } & ,0015491906 & \\
\hline & Skewness & &, 013 & ,156 \\
\hline & Kurtosis & &, 734 & ,310 \\
\hline 103,104 & Mean & &,- 000077199 &, 0000789636 \\
\hline & $95 \%$ Confidence Interval for & Lower Bound &,- 000232736 & \\
\hline & Mean & Upper Bound &, 0000783380 & \\
\hline & $5 \%$ Trimmed Mean & &,- 000080144 & \\
\hline & Median & &,- 000072547 & \\
\hline & Variance & &, 000 & \\
\hline & Std. Deviation & & ,0012359752 & \\
\hline & Minimum & &,- 004995261 & \\
\hline & Maximum & & ,0033251570 & \\
\hline
\end{tabular}


Y AXIS Descriptives

\begin{tabular}{|c|c|c|c|c|}
\hline & & & Statistic & Std. Error \\
\hline & \multicolumn{2}{|l|}{ Range } & ,0083204183 & \\
\hline & \multicolumn{2}{|l|}{ Interquartile Range } & ,0016687605 & \\
\hline & \multicolumn{2}{|l|}{ Skewness } &,- 084 & , 156 \\
\hline & \multicolumn{2}{|l|}{ Kurtosis } & ,866 & ,310 \\
\hline \multirow[t]{13}{*}{105,106} & \multicolumn{2}{|l|}{ Mean } &,- 000306255 & ,0000888729 \\
\hline & \multirow{2}{*}{$\begin{array}{l}95 \% \text { Confidence Interval for } \\
\text { Mean }\end{array}$} & Lower Bound &,- 000481311 & \\
\hline & & Upper Bound &,- 000131199 & \\
\hline & \multicolumn{2}{|l|}{$5 \%$ Trimmed Mean } &,- 000309070 & \\
\hline & \multicolumn{2}{|l|}{ Median } &,- 000327404 & \\
\hline & \multicolumn{2}{|l|}{ Variance } & ,000 & \\
\hline & \multicolumn{2}{|l|}{ Std. Deviation } & ,0013910814 & \\
\hline & \multicolumn{2}{|l|}{ Minimum } &,- 005622719 & \\
\hline & \multicolumn{2}{|l|}{ Maximum } & ,0035351289 & \\
\hline & \multicolumn{2}{|l|}{ Range } & ,0091578480 & \\
\hline & \multicolumn{2}{|l|}{ Interquartile Range } & ,0018152810 & \\
\hline & \multicolumn{2}{|l|}{ Skewness } &,- 074 & , 156 \\
\hline & \multicolumn{2}{|l|}{ Kurtosis } & ,833 & ,310 \\
\hline
\end{tabular}

Y AXIS Tests of Normality

\begin{tabular}{|cc|c|c|c|c|c} 
& \multicolumn{2}{c}{ Kolmogorov-Smirnov } & \multicolumn{3}{c}{ Shapiro-Wilk } \\
\hline & Statistic & df & Sig. & Statistic & df & Sig. \\
\hline 11,12 &, 043 & 245 &, $200^{*}$ &, 996 & 245 &, 815 \\
\hline 13,14 &, 036 & 245 &, $200^{*}$ &, 996 & 245 &, 712 \\
\hline 15,16 &, 038 & 245 &, $200^{*}$ &, 996 & 245 &, 734 \\
\hline 17,18 &, 029 & 245 &, $200^{*}$ &, 996 & 245 &, 698 \\
\hline 19,20 &, 031 & 245 &, $200^{*}$ &, 996 & 245 &, 704 \\
\hline 21,22 &, 055 & 245 &, 068 &, 993 & 245 &, 325 \\
\hline 23,24 &, 048 & 245 &, $200^{*}$ &, 996 & 245 &, 788 \\
\hline 25,26 &, 066 & 245 &, 012 &, 994 & 245 &, 464 \\
\hline 27,28 &, 064 & 245 &, 017 &, 994 & 245 &, 396 \\
\hline 29,30 &, 037 & 245 &, $200^{*}$ &, 996 & 245 &, 794 \\
\hline 31,32 &, 036 & 245 &, $200^{*}$ &, 996 & 245 &, 752 \\
\hline 33,34 &, 036 & 245 &, $200^{*}$ &, 995 & 245 &, 536 \\
\hline 35,36 &, 023 & 245 &, $200^{*}$ &, 996 & 245 &, 771 \\
\hline 37,38 &, 064 & 245 &, 018 &, 986 & 245 &, 014 \\
\hline 39,40 &, 045 & 245 &, $200^{*}$ &, 991 & 245 &, 144 \\
\hline
\end{tabular}


Y AXIS Tests of Normality

\begin{tabular}{|c|c|c|c|c|c|c|}
\hline & \multicolumn{3}{|c|}{ Kolmogorov-Smirnov ${ }^{a}$} & \multicolumn{3}{|c|}{ Shapiro-Wilk } \\
\hline & Statistic & $d f$ & Sig. & Statistic & $\mathrm{df}$ & Sig. \\
\hline 41,42 & ,037 & 245 &, $200^{*}$ & ,993 & 245 & ,290 \\
\hline 43,44 & ,028 & 245 & ,200* & ,997 & 245 & ,873 \\
\hline 45,46 & ,046 & 245 &, $200^{*}$ & ,988 & 245 & ,045 \\
\hline 47,48 & ,021 & 245 &, $200^{*}$ & ,998 & 245 & ,983 \\
\hline 49,50 & ,045 & 245 & ,200* & ,994 & 245 &, 514 \\
\hline 51,52 &, 047 & 245 &, $200^{*}$ & ,995 & 245 & ,648 \\
\hline 53,54 & 041 & 245 &, $200^{*}$ & ,996 & 245 & ,704 \\
\hline 55,56 & ,036 & 245 & ,200* & ,994 & 245 & ,433 \\
\hline 57,58 & ,042 & 245 &, $200^{*}$ & ,993 & 245 & ,342 \\
\hline 59,60 &, 036 & 245 &, $200^{*}$ & ,996 & 245 & ,771 \\
\hline 61,62 & ,042 & 245 &, $200^{*}$ & ,990 & 245 & ,073 \\
\hline 63,64 & ,053 & 245 & ,092 & ,988 & 245 & ,046 \\
\hline 65,66 & ,053 & 245 & ,087 & ,991 & 245 & ,163 \\
\hline 67,68 & ,045 & 245 & ,200* & ,994 & 245 & ,495 \\
\hline 69,70 & ,038 & 245 &, $200^{*}$ & ,993 & 245 & ,358 \\
\hline 71,72 & ,049 & 245 &, $200^{*}$ & ,995 & 245 & ,647 \\
\hline 73,74 & ,052 & 245 &, $200^{*}$ & ,991 & 245 & ,154 \\
\hline 75,76 & ,035 & 245 &, $200^{*}$ & ,993 & 245 & ,316 \\
\hline 77,78 & ,039 & 245 & ,200* & ,995 & 245 &, 569 \\
\hline 79,80 & ,039 & 245 &, $200^{*}$ & ,995 & 245 &, 569 \\
\hline 81,82 & ,049 & 245 &, $200^{*}$ & ,995 & 245 &, 571 \\
\hline 83,84 & ,029 & 245 &, $200^{*}$ & ,997 & 245 & ,916 \\
\hline 85,86 & ,040 & 245 &, $200^{*}$ & ,996 & 245 & ,852 \\
\hline 87,88 & ,048 & 245 & ,200* & ,996 & 245 & ,783 \\
\hline 89,90 & ,046 & 245 & ,200* & ,994 & 245 & ,446 \\
\hline 91,92 & ,045 & 245 &, $200^{*}$ & ,996 & 245 & ,821 \\
\hline 93,94 & ,042 & 245 & ,200* & ,990 & 245 & ,081 \\
\hline 95,96 & ,039 & 245 & ,200* & ,995 & 245 & ,648 \\
\hline 97,98 & ,039 & 245 & ,200* & ,995 & 245 & ,609 \\
\hline 99,100 &, 044 & 245 &, $200^{*}$ & ,989 & 245 & ,062 \\
\hline 101,102 & ,043 & 245 & ,200* & ,992 & 245 & ,213 \\
\hline 103,104 & ,040 & 245 &, $200^{*}$ & ,990 & 245 & ,107 \\
\hline 105,106 & ,036 & 245 &, $200^{*}$ & ,992 & 245 & ,177 \\
\hline
\end{tabular}


*. This is a lower bound of the true significance.

a. Lilliefors Significance Correction

Página 37 
X AXIS One-Sample Test

\begin{tabular}{|c|c|c|c|c|c|c|}
\hline & \multicolumn{6}{|c|}{ Test Value $=0$} \\
\hline & \multirow[b]{2}{*}{$\mathrm{t}$} & \multirow[b]{2}{*}{ df } & \multirow[b]{2}{*}{ Sig. (2-tailed) } & \multirow{2}{*}{$\begin{array}{c}\text { Mean } \\
\text { Difference }\end{array}$} & \multicolumn{2}{|c|}{$\begin{array}{c}\text { 95\% Confidence Interval of the } \\
\text { Difference }\end{array}$} \\
\hline & & & & & Lower & Upper \\
\hline 11,12 & 3,972 & 244 &, 000 & ,0002313175 & ,0001166144 &, 0003460205 \\
\hline 13,14 & 3,568 & 244 &, 000 & ,0001915536 & ,0000858075 & ,0002972997 \\
\hline 15,16 & 2,924 & 244 & ,004 & ,0001462272 & ,0000477379 & ,0002447164 \\
\hline 17,18 & 2,591 & 244 &, 010 & ,0001248311 & ,0000299213 & ,0002197409 \\
\hline 19,20 & 2,748 & 244 & ,006 & ,0001310097 & ,0000371176 & ,0002249017 \\
\hline 21,22 & 2,842 & 244 & ,005 & ,0001433855 & ,0000439941 & ,0002427770 \\
\hline 23,24 & 3,911 & 244 & ,000 & ,0002096760 & ,0001040750 & ,0003152771 \\
\hline 25,26 & 4,975 & 244 &, 000 & ,0002945792 & ,0001779358 & ,0004112225 \\
\hline 27,28 & 5,820 & 244 &, 000 &, 0003601511 &, 0002382528 & ,0004820493 \\
\hline 29,30 & 6,797 & 244 & ,000 & ,0004260654 & ,0003025998 & ,0005495309 \\
\hline 31,32 & 7,966 & 244 &, 000 & ,0005016958 & ,0003776365 &, 0006257550 \\
\hline 33,34 & 8,665 & 244 & ,000 & ,0005107779 & ,0003946717 & ,0006268840 \\
\hline 35,36 & 9,171 & 244 & ,000 & ,0005099598 & ,0004004350 & ,0006194846 \\
\hline 37,38 & 8,916 & 244 & ,000 & ,0004126229 & ,0003214695 & ,0005037763 \\
\hline 39,40 & 8,304 & 244 &, 000 &, 0002941511 & ,0002243751 &, 0003639272 \\
\hline 41,42 & 6,827 & 244 &, 000 & ,0001382077 & ,0000983343 &, 0001780810 \\
\hline 43,44 & $-2,130$ & 244 & ,034 &,- 000227620 &,- 000438101 &,- 000017139 \\
\hline 45,46 & $-2,905$ & 244 &, 004 &,- 000249842 &,- 000419255 &,- 000080428 \\
\hline 47,48 & $-2,955$ & 244 &, 003 &,- 000240486 &,- 000400814 &,- 000080158 \\
\hline 49,50 & $-1,797$ & 244 & ,074 &,- 000131140 &,- 000274860 & ,0000125798 \\
\hline 51,52 & ,385 & 244 & ,701 & ,0000275336 &,- 000113500 & ,0001685675 \\
\hline 53,54 & ,631 & 244 &, 529 &, 0000435064 &,- 000092392 &, 0001794053 \\
\hline 55,56 & $-1,721$ & 244 &, 086 &,- 000105380 &,- 000225979 & ,0000152204 \\
\hline 57,58 & $-2,910$ & 244 & ,004 &,- 000223161 &,- 000374202 &,- 000072120 \\
\hline 59,60 & $-3,095$ & 244 &, 002 &,- 000269791 &,- 000441517 &,- 000098066 \\
\hline 61,62 & $-3,451$ & 244 &, 001 &,- 000220828 &,- 000346873 &,- 000094783 \\
\hline 63,64 & 1,396 & 244 &, 164 & ,0000840169 &,- 000034537 & ,0002025709 \\
\hline 65,66 & 4,095 & 244 &, 000 & ,0002309626 & ,0001198580 &, 0003420672 \\
\hline 67,68 & 1,740 & 244 &, 083 & ,0000808358 &,- 000010670 &, 0001723413 \\
\hline 69,70 & $-3,661$ & 244 &, 000 &,- 000144087 &,- 000221604 &,- 000066571 \\
\hline 71,72 & ,474 & 244 & ,636 & ,0000221871 &,- 000070108 & ,0001144824 \\
\hline 73,74 & 1,876 & 244 & ,062 & ,0001005493 &,- 000005028 & ,0002061266 \\
\hline 75,76 &,- 086 & 244 & ,931 &,- 000004876 &,- 000115974 &, 0001062220 \\
\hline 77,78 & 4,185 & 244 & ,000 & ,0002258512 & ,0001195487 & ,0003321538 \\
\hline 79,80 & 4,185 & 244 & ,000 & ,0002258512 & ,0001195487 & ,0003321538 \\
\hline
\end{tabular}

Página 1 
X AXIS One-Sample Test

\begin{tabular}{|c|c|c|c|c|c|c|}
\hline & \multicolumn{6}{|c|}{ Test Value $=0$} \\
\hline & \multirow[b]{2}{*}{$\mathrm{t}$} & \multirow[b]{2}{*}{ df } & \multirow[b]{2}{*}{ Sig. (2-tailed) } & \multirow{2}{*}{$\begin{array}{c}\text { Mean } \\
\text { Difference }\end{array}$} & \multicolumn{2}{|c|}{$\begin{array}{l}\text { 95\% Confidence Interval of the } \\
\text { Difference }\end{array}$} \\
\hline & & & & & Lower & Upper \\
\hline 81,82 & $-2,947$ & 244 & ,004 &,- 000055546 &,- 000092670 &,- 000018422 \\
\hline 83,84 & $-8,567$ & 244 &, 000 &,- 000184536 &,- 000226968 &,- 000142105 \\
\hline 85,86 & $-7,465$ & 244 & ,000 &,- 000220985 &,- 000279294 &,- 000162677 \\
\hline 87,88 & $-9,051$ & 244 & ,000 &,- 000184325 &,- 000224437 &,- 000144213 \\
\hline 89,90 & $-9,102$ & 244 & ,000 &,- 000141736 &,- 000172407 &,- 000111064 \\
\hline 91,92 & 3,928 & 244 & ,000 & ,0002124531 & 0001059173, & ,0003189889 \\
\hline 93,94 & 3,804 & 244 & ,000 & 0001298403, & ,0000626099 & ,0001970708 \\
\hline 95,96 & 4,131 & 244 &, 000 & 0001933900, & ,0001011727 & ,0002856073 \\
\hline 97,98 & 3,238 & 244 & ,001 & 0000457027, & ,0000178967 & ,0000735086 \\
\hline 99,100 & 2,953 & 244 & ,003 & ,0000819929 & ,0000272980 & ,0001366878 \\
\hline 101,102 & 3,535 & 244 & ,000 & 0001412914, & ,0000625725 & ,0002200103 \\
\hline 103,104 & 2,830 & 244 & ,005 & 0000807301, & ,0000245307 & ,0001369295 \\
\hline 105,106 & 2,099 & 244 & ,037 & ,0000508855 & ,0000031336 &, 0000986374 \\
\hline
\end{tabular}

Y AXIS One-Sample Test

\begin{tabular}{|c|c|c|c|c|c|c|}
\hline & \multicolumn{6}{|c|}{ Test Value $=0$} \\
\hline & \multirow[b]{2}{*}{$t$} & \multirow[b]{2}{*}{ df } & \multirow[b]{2}{*}{ Sig. (2-tailed) } & \multirow{2}{*}{$\begin{array}{c}\text { Mean } \\
\text { Difference }\end{array}$} & \multicolumn{2}{|c|}{$\begin{array}{c}\text { 95\% Confidence Interval of the } \\
\text { Difference }\end{array}$} \\
\hline & & & & & Lower & Upper \\
\hline 11,12 & $-1,961$ & 244 & ,051 &,- 000244683 &,- 000490491 & ,0000011244 \\
\hline 13,14 & $-2,373$ & 244 &, 018 &,- 000295515 &,- 000540812 &,- 000050219 \\
\hline 15,16 & $-2,675$ & 244 &, 008 &,- 000327807 &,- 000569152 &,- 000086461 \\
\hline 17,18 & $-2,960$ & 244 & ,003 &,- 000359580 &,- 000598825 &,- 000120334 \\
\hline 19,20 & $-2,757$ & 244 & ,006 &,- 000334691 &,- 000573822 &,- 000095559 \\
\hline 21,22 & $-2,343$ & 244 & ,020 &,- 000284392 &,- 000523522 &,- 000045262 \\
\hline 23,24 & $-1,666$ & 244 & 097 &,- 000200313 &,- 000437096 & ,0000364705 \\
\hline 25,26 &,- 725 & 244 & ,469 &,- 000083471 &,- 000310224 & ,0001432833 \\
\hline 27,28 & ,152 & 244 & ,879 & ,0000161071 &,- 000192817 & ,0002250311 \\
\hline 29,30 & 1,383 & 244 & , 168 & ,0001288098 &,- 000054670 & ,0003122900 \\
\hline 31,32 & 2,565 & 244 & 011 & ,0002022773 & ,0000469525 & ,0003576022 \\
\hline 33,34 & 4,521 & 244 &, 000 & ,0002932439 & ,0001654813 & ,0004210065 \\
\hline 35,36 & 5,414 & 244 &, 000 & ,0003036773 & ,0001931937 & ,0004141609 \\
\hline 37,38 & 5,892 & 244 &, 000 & ,0003272164 & ,0002178251 & ,0004366076 \\
\hline 39,40 & 5,266 & 244 &, 000 & ,0003276207 & ,0002050659 &, 0004501756 \\
\hline 41,42 & 5,002 & 244 & ,000 & 0003674284 &, 0002227441 &, 0005121128 \\
\hline
\end{tabular}

Página 2 
Y AXIS One-Sample Test

\begin{tabular}{|c|c|c|c|c|c|c|}
\hline & \multicolumn{6}{|c|}{ Test Value $=0$} \\
\hline & \multirow[b]{2}{*}{$\mathrm{t}$} & \multirow[b]{2}{*}{ df } & \multirow[b]{2}{*}{ Sig. (2-tailed) } & \multirow{2}{*}{$\begin{array}{c}\text { Mean } \\
\text { Difference }\end{array}$} & \multicolumn{2}{|c|}{$\begin{array}{l}\text { 95\% Confidence Interval of the } \\
\text { Difference }\end{array}$} \\
\hline & & & & & Lower & Upper \\
\hline 43,44 & $-2,935$ & 244 &, 004 &,- 000237104 &,- 000396210 &,- 000077999 \\
\hline 45,46 & $-2,384$ & 244 & ,018 &,- 000185294 &,- 000338376 &,- 000032212 \\
\hline 47,48 & $-3,349$ & 244 & 001 &,- 000259171 &,- 000411619 &,- 000106723 \\
\hline 49,50 & $-3,526$ & 244 & 001 &,- 000304502 &,- 000474619 &,- 000134386 \\
\hline 51,52 & $-1,682$ & 244 & ,094 &,- 000166892 &,- 000362334 & ,0000285506 \\
\hline 53,54 &,- 863 & 244 & ,389 &,- 000083900 &,- 000275348 & ,0001075473 \\
\hline 55,56 & $-2,310$ & 244 & 022 &,- 000189767 &,- 000351589 &,- 000027944 \\
\hline 57,58 & $-2,704$ & 244 &, 007 &,- 000195194 &,- 000337408 &,- 000052981 \\
\hline 59,60 & $-3,562$ & 244 &, 000 &,- 000246237 &,- 000382388 &,- 000110086 \\
\hline 61,62 & 2,388 & 244 & ,018 & ,0001321100 & ,0000231354 & ,0002410846 \\
\hline 63,64 & 2,725 & 244 &, 007 & 0001428781 & ,0000395815 & ,0002461748 \\
\hline 65,66 & 4,075 & 244 &, 000 & ,0002019674 & ,0001043388 & ,0002995959 \\
\hline 67,68 & 3,750 & 244 &, 000 & ,0001854673 & ,0000880535 & ,0002828812 \\
\hline 69,70 & , 450 & 244 & ,653 & ,0000238691 &,- 000080531 & ,0001282694 \\
\hline 71,72 & 1,877 & 244 & ,062 & ,0000967947 &,- 000004800 & ,0001983892 \\
\hline 73,74 & 3,450 & 244 & 001 & ,0001845866 & ,0000792112 & ,0002899620 \\
\hline 75,76 & 3,903 & 244 & ,000 & ,0002182225 & ,0001081038 & ,0003283411 \\
\hline 77,78 & 6,839 & 244 & ,000 & ,0004086501 & ,0002909514 & ,0005263488 \\
\hline 79,80 & 6,839 & 244 &, 000 & ,0004086501 & ,0002909514 & ,0005263488 \\
\hline 81,82 & $-2,944$ & 244 & ,004 &,- 000190197 &,- 000317451 &,- 000062943 \\
\hline 83,84 & 2,560 & 244 & ,011 & ,0001946568 & ,0000448568 & ,0003444568 \\
\hline 85,86 & 4,310 & 244 &, 000 & ,0003148154 & 0001709559, & ,0004586749 \\
\hline 87,88 & 3,441 & 244 & ,001 & ,0002925784 & 0001250987, & ,0004600582 \\
\hline 89,90 & 2,812 & 244 & ,005 & ,0002815765 & ,0000843468 & ,0004788061 \\
\hline 91,92 & 2,652 & 244 & ,009 & ,0002322817 & ,0000597645 & 0004047989, \\
\hline 93,94 &,- 703 & 244 & ,483 &,- 000054863 &,- 000208566 & 0000988399, \\
\hline 95,96 & 2,347 & 244 & ,020 & ,0001884256 & ,0000302783 & ,0003465728 \\
\hline 97,98 &,- 975 & 244 & ,331 &,- 000096210 &,- 000290666 & ,0000982447, \\
\hline 99,100 & $-1,033$ & 244 & ,303 &,- 000079174 &,- 000230140 & 0000717915, \\
\hline 101,102 & $-1,222$ & 244 & ,223 &,- 000096588 &,- 000252315 & 0000591397, \\
\hline 103,104 &,- 978 & 244 & ,329 &,- 000077199 &,- 000232736 & 0000783380, \\
\hline 105,106 & $-3,446$ & 244 & ,001 &,- 000306255 &,- 000481311 &,- 000131199 \\
\hline
\end{tabular}

Página 3 